\title{
Liquid Etherification of Alkoxybenzyl Alcohols, Cinnamyl Alcohols and Fluorinated Phenylalkenols with Platinum on Carbon (Pt/C) and Palladium on Carbon (Pd/C)
}

\author{
Thies Thiemann \\ Department of Chemistry, College of Science, United Arab Emirates University, Al Ain, Abu Dhabi, UAE \\ Email: hies@uaeu.ac.ae
}

How to cite this paper: Thiemann, T. (2018) Liquid Etherification of Alkoxybenzyl Alcohols, Cinnamyl Alcohols and Fluorinated Phenylalkenols with Platinum on Carbon $(\mathrm{Pt} / \mathrm{C})$ and Palladium on Carbon $(\mathrm{Pd} / \mathrm{C})$. International Journal of Organic Chemistry, 8, 84-104.

https://doi.org/10.4236/ijoc.2018.81005

Received: January 18, 2018

Accepted: March 3, 2018

Published: March 6, 2018

Copyright $\odot 2018$ by author and Scientific Research Publishing Inc. This work is licensed under the Creative Commons Attribution International License (CC BY 4.0).

http://creativecommons.org/licenses/by/4.0/

\begin{abstract}
In the presence of either $\mathrm{Pd} / \mathrm{C}$ or $\mathrm{Pt} / \mathrm{C}$, 4-alkoxybenzyl alcohols, 4-alkoxyphenylalkyl alcohols and cinnamyl alcohols undergo liquid etherification to bis(4-alkoxybenzyl) ethers, bis[(4-alkoxybenzyl)alkyl] ethers and bis(cinnamyl) ethers at temperatures of $100^{\circ} \mathrm{C}-135^{\circ} \mathrm{C}$. In a number of cases, $\mathrm{Pt} / \mathrm{C}$ and $\mathrm{Pd} / \mathrm{C}$ as catalysts, also when they are doped with $\mathrm{Ru}$, show distinct differences when the reactions run in air, with an oxidative dehydrogenation of the alcohols as side-reaction, yielding the corresponding ketones and aldehydes as by-products. Also, the reactivity of fluorinated phenylalkenols under these conditions has been investigated. Furthermore, the immobilization of alkyl aryl ethers on silica within a column with passage of hexane as solvent was found to lead to the formation and elution of styrenes from the column in fair yield.
\end{abstract}

\section{Keywords}

Benzyl Alcohols, Etherification, Platinum on Carbon, Palladium on Carbon, Activated Carbon, Solvent Free Reaction

\section{Introduction}

Bis[(4-alkoxybenzyl)alkyl] ethers are interesting substrates for the preparation of styrene derivatives as useful building blocks in polymers [1]. For this reason, the author sought a convenient reaction system to prepare the acid sensitive bis[(4-alkoxybenzyl)alkyl] ethers. Because of environmental concerns, the use of reaction solvents was to be restricted. 
Usually, benzyl ethers are prepared by reaction of an activated benzyl reagent such as a benzyl halide, benzyl tosylate or other reactive benzyl ester with an alcohol component [2]. It is more economical, however, to prepare at least symmetric bis(benzyl) ethers directly from two molecules of the corresponding benzyl alcohol. Latter reactions are known, where Lewis acid catalysts such as $\mathrm{ZnCl}_{2}$ [3], $\mathrm{Mg}\left(\mathrm{ClO}_{4}\right)_{2}$ [4] and aluminum dodecatungstophosphate $\left(\mathrm{AlPW}_{12} \mathrm{O}_{40}\right)$ [5] as well as $\mathrm{MeAl}\left[\mathrm{N}\left(\mathrm{SO}_{2} \mathrm{CF}_{3}\right)_{2}\right]_{2}[6]$ have been used. Some of the catalysts are incompatible with bis[(4-alkoxybenzyl)alkyl] ethers such as $\mathrm{ZnCl}_{2}$ or less readily accessible $\left(\mathrm{AlPW}_{12} \mathrm{O}_{40}, \mathrm{MeAl}\left[\mathrm{N}\left(\mathrm{SO}_{2} \mathrm{CF}_{3}\right)_{2}\right]_{2}\right)$. Bentonite clays have been used successfully as catalyst [7] [8], although product mixtures have been described for certain substrates [8], as here aromatic substitution reactions are catalyzed, also. It is interesting to note that bis(benzyl) ethers have been prepared by simple heating of the benzylic alcohols in DMSO at $175^{\circ} \mathrm{C}$ [9]. Our experience is, however, that benzyl alkyl alcohols are prone to oxidize under these conditions. Additional by-products stem from the dehydration of the alcohols under these conditions.

Recently, it has been realized that $\mathrm{Pd}(\mathrm{II})$ species catalyze the etherification of benzyl alcohols, where etherification reactions with various alcohols have been carried out with $\mathrm{Pd}(\mathrm{II})$ catalysts [10] [11] [12] [13] in nitromethane [10] and with $\mathrm{Pd}(\mathrm{Cl})_{2}\left(\mathrm{CH}_{3} \mathrm{CN}\right)_{2}$ in dichloroethane [11].

In the following, the author shows that also $\mathrm{Pd}(0)$ and $\mathrm{Pt}(0)$ pro-catalysts [14] [15], which are less expensive than $\mathrm{Pd}(\mathrm{II})$ complexes and which are also recyclable, can be used for the etherification of benzyl alcohols in solvent free systems. There has been one prior report of the use of $\operatorname{Pd}(0)$ in ionic liquids to prepare dibenzyl ethers from alcohols [16]. In addition, it is shown that the obtained ethers can be good starting materials for substituted styrenes, when adsorbed to silica gel, where the alkene products are eluted continuously with hexane.

\section{Experimental}

General-Melting points were measured on a Yanaco microscopic hotstage and are uncorrected. Infrared spectra were measured with JASCO IR-700 and Nippon Denshi JIR-AQ2OM instruments. ${ }^{1} \mathrm{H}$ and ${ }^{13} \mathrm{C}$ NMR spectra were recorded with a JEOL EX-270 spectrometer $\left({ }^{1} \mathrm{H}\right.$ at $270 \mathrm{MHz},{ }^{13} \mathrm{C}$ at $\left.67.8 \mathrm{MHz}\right)$. The assignment of the 13C-NMR spectra was aided by DEPT (Distortionless Enhancement by Polarization Transfer) technique. The chemical shifts are relative to TMS (solvent $\mathrm{CDCl}_{3}$, unless otherwise noted). Mass spectra were measured with a JMS-01-SG-2 spectrometer. Column chromatography was carried out on Wakogel 300.

Chemicals and Reagents-Platinum on activated carbon (5 w\%; Wako [167-13,911]), palladium on activated carbon (10 w\%; Kishida [400-59,092]), Pearlman's catalyst $\left(20 \mathrm{w} \% \mathrm{Pd}(\mathrm{OH})_{2}\right.$ [dry weight] on carbon, $<50 \%$ moisture, Aldrich [21,291-1]) and Lindlar catalyst (5 w\% $\mathrm{Pd}$ on $\mathrm{CaCO}_{3}$, poisoned with $\mathrm{Pb}$, Aldrich $[205,737])$ were acquired commercially. Patinum-ruthenium catalyst on 
carbon nanofiber (Pt-Ru CNF) was prepared according to the literature [17]. Phenol (4) (Kishida), anisole (5b) (Kishida), and propionyl chloride (CICA) were purchased. 4-Dodecyloxybenzene (5a) was prepared by PTC-catalyzed etherification of phenol (4) (see below, Scheme 1) [18]. 3,4-Dimethoxybenzaldehyde (2d) (Kishida), 4-tolualdehyde (2e) (TCI), 2-ethoxybenzaldehyde (2c) (TCI), 4-hydrox-ybenzaldehyde (1) (TCI), 4-methoxyacetophenone (8a) (Aldrich), cinnamaldehyde (10b) (Kishida) and 4-methoxybenzylalcohol (3e) (Wako) were obtained commercially. $D L$-1-(4-alkoxyphenyl)propyl alcohols 7 were obtained from the corresponding ketones 6 by carbonyl reduction with $\mathrm{NaBH}_{4}$ in methanol (Scheme 1). 4-Alkoxybenzaldehydes 2 were prepared from 4-hydroxybenzaldehyde (1) by etherification with alkyl iodides (KOH, DMSO) [19] (Scheme 1). Phenylalkanones 6 were prepared via acylation alkoxybenzenes (5) with propionyl chloride ( $\mathrm{AlCl}_{3}, 1,2$-dichloroethane). These were reduced to alcohols $7\left(\mathrm{NaBH}_{4}, \mathrm{MeOH}\right)$ (Figure 1). The alkoxybenzenes 5 used were either obtained by PTC catalyzed or by $\mathrm{KOH} / \mathrm{DMSO}$ mediated $O$-alkylation of phenol (Scheme 1). A typical example of the sequence can be found below with the synthesis of DL-(4-dodecyloxyphenyl)propyl alcohol (7a) (Scheme 1). Reduction of 4-alkoxybenzaldehydes 2 gave benzyl alcohols $3\left(\mathrm{NaBH}_{4}, \mathrm{MeOH}\right.$ or $\mathrm{LiAlH}_{4}, \mathrm{THF}$ ) (Scheme 2). (E)-4-(3,4,5-Trifluorophenyl)-but-3-en-2-one (14a) and E)-4-(2,4-difluoro)but-3-en-2-one (14b) were prepared by solvent free Wittig reaction of 3,4,5-trifluorobenzaldehyde (12a)/2,4-difluorobenzaldehyde (12b) and acetylmethylidenetriphenylphosphorane (13) [20], respectively, with subsequent Luche reduction (see below, Scheme 4). Cinnamyl alcohols 11 were prepared by Wittig olefination of the corresponding benzaldehydes 2 , using a minimal amount of chloroform as solvent [21] [22] and subsequent reduction of the cinnamaldehydes10 with $\mathrm{NaBH}_{4}$ in $\mathrm{MeOH}$ in the presence of $\mathrm{CeCl}_{3}$ (Luche reduction, Scheme 3) [23]. Benzyhydrols 17 were prepared from the commercially available benzophenones 18a and $\mathbf{1 8 b}$ (Aldrich) by reduction with $\mathrm{NaBH}_{4}$ in $\mathrm{MeOH}$

(E)-4-(2,4-Diffuoro) but-3-en-2-one (14b).-2,4-Difluorobenzaldehyde (12b, $1.0 \mathrm{~g}, 7.0 \mathrm{mmol})$ and $13(3.58 \mathrm{~g}, 11.2 \mathrm{mmol})$ were reacted at $100^{\circ} \mathrm{C}$ for $40 \mathrm{~min}$.

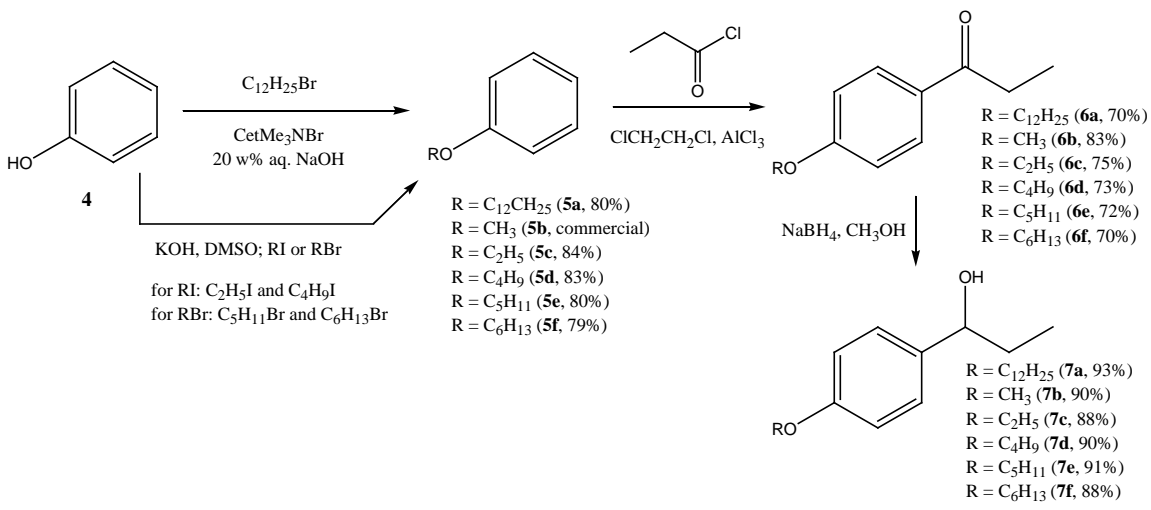

Scheme 1. Preparation of benzyl alcohols 3 as substrates. 

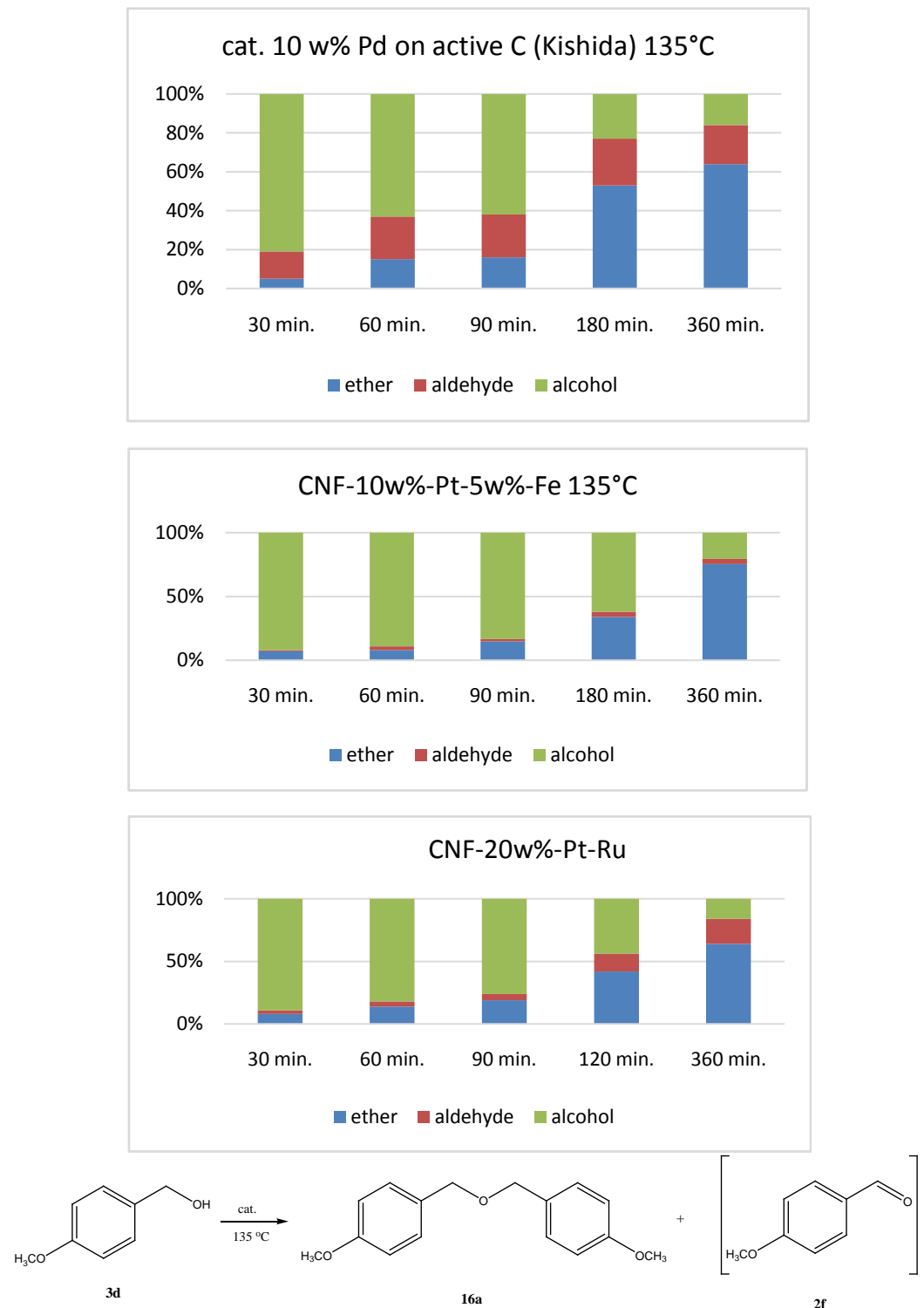

Figure 1. Solventless ether formation of benzyl alcohol 3d over different Pt- and Pd-catalysts.

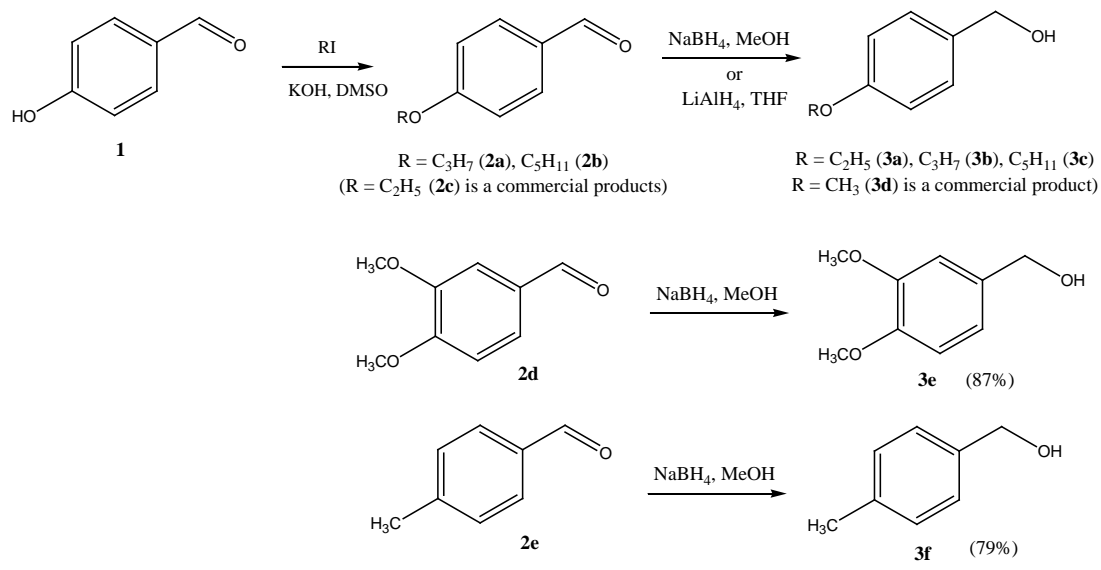

Scheme 2. Preparation of phenylalkanols 7. 


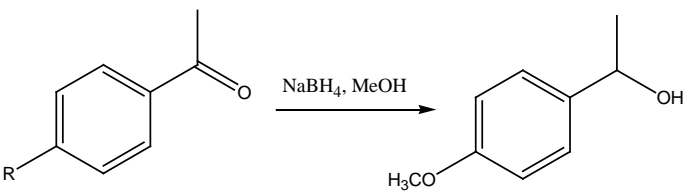

$\mathrm{R}=\mathrm{OCH}_{3}(\mathbf{8 a}), \mathrm{CH}_{3}(\mathbf{8 b})$

$\mathrm{R}=\mathrm{OCH}_{3}(\mathbf{9 a}, 87 \%), \mathrm{R}=\mathrm{CH}_{3}(\mathbf{9 b}, 85 \%)$

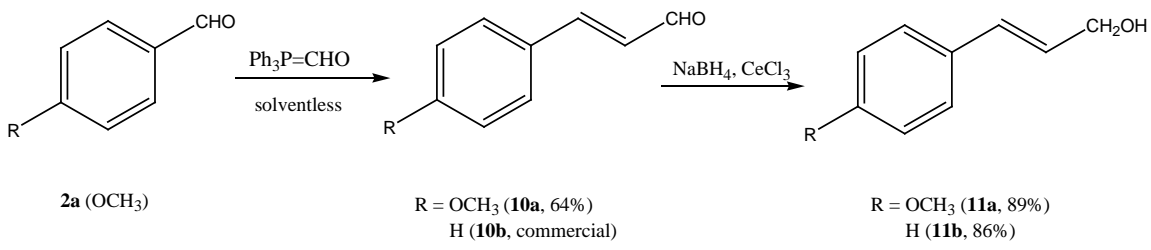

Scheme 3. Preparation of 1-arylethanols 9 and cinnamyl alcohols 11.

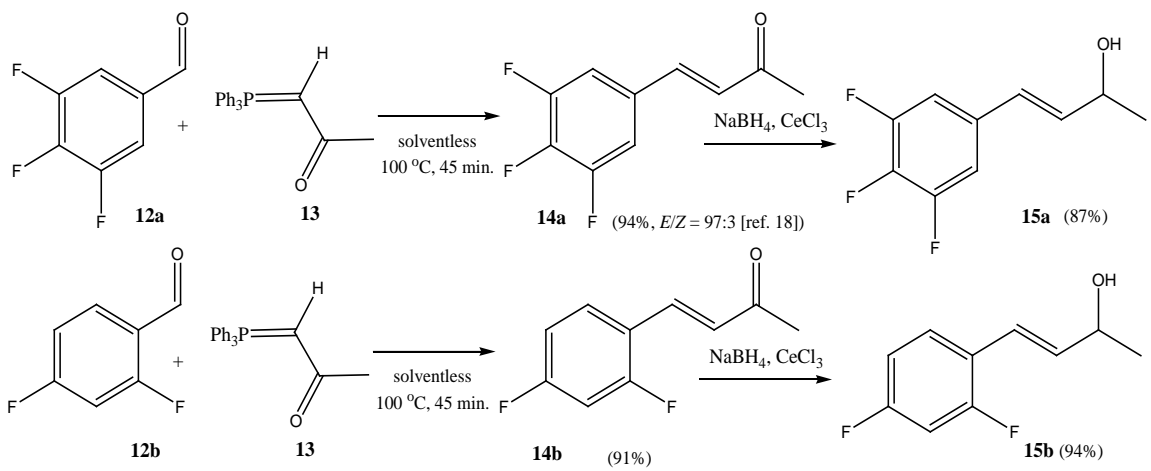

Scheme 4. Preparation of fluorophenylbut-3-en-2-ols 15

Column chromatography on silica gel (hexane/ether/ $\mathrm{CHCl}_{3}$ 4:1:1) gave $(E)-14 \mathrm{~b}$ $(1.2 \mathrm{~g}, 94 \%)$ as a colorless oil; IR (neat) $v 2924,1674(\mathrm{C}=\mathrm{O}), 1620,1500,1430$, 1360, 1255, 1177, 1142, 1090, 968, 910, 851, 808, $729 \mathrm{~cm}^{-1}$; ${ }^{1} \mathrm{H}-\mathrm{NMR}(270 \mathrm{MHz}$, $\left.\mathrm{CDCl}_{3}\right) \delta 2.39\left(\mathrm{~s}, 3 \mathrm{H}, \mathrm{CH}_{3}\right), 6.73\left(\mathrm{~d}, 1 \mathrm{H},{ }^{3} J=16.5 \mathrm{~Hz}\right), 6.93(\mathrm{~m}, 2 \mathrm{H}), 7.55(\mathrm{~m}$, $1 \mathrm{H}), 7.60\left(\mathrm{~d}, 1 \mathrm{H},{ }^{3} J=16.5 \mathrm{~Hz}\right) ;{ }^{13} \mathrm{C}-\mathrm{NMR}\left(67.8 \mathrm{MHz}, \mathrm{CDCl}_{3}\right) \delta 27.5\left(\mathrm{CH}_{3}\right), 104.6$ $\left(\mathrm{dd}, J_{\mathrm{CF}}=25.8 \mathrm{~Hz}, J_{\mathrm{CF}}=25.8 \mathrm{~Hz}\right), 112.2\left(\mathrm{dd}, J_{\mathrm{CF}}=21.7 \mathrm{~Hz}, J_{\mathrm{CF}}=3.3 \mathrm{~Hz}\right), 119.0$ $\left(\mathrm{dd}, J_{\mathrm{CF}}=11.8 \mathrm{~Hz}, J_{\mathrm{CF}}=3.9 \mathrm{~Hz}\right), 128.8\left(\mathrm{dd}, J_{\mathrm{CF}}=5.0 \mathrm{~Hz}, J_{\mathrm{CF}}=2.2 \mathrm{~Hz}\right), 129.9(\mathrm{dd}$, $\left.J_{\mathrm{CF}}=9.5 \mathrm{~Hz}, J_{\mathrm{CF}}=4.5 \mathrm{~Hz}\right), 134.6\left(\mathrm{dd}, J_{\mathrm{CF}}=2.8 \mathrm{~Hz}, J_{\mathrm{CF}}=1.7 \mathrm{~Hz}\right), 161.6\left(\mathrm{dd}, J_{\mathrm{CF}}=\right.$ $\left.-256 \mathrm{~Hz}, J_{\mathrm{CF}}=12.3 \mathrm{~Hz}\right), 164.1\left(\mathrm{dd}, J_{\mathrm{CF}}=-254 \mathrm{~Hz}, J_{\mathrm{CF}}=12.3 \mathrm{~Hz}\right), 190.1(\mathrm{CO}) ; \mathrm{MS}$ (EI, $70 \mathrm{eV}) \mathrm{m} / z(\%) 182\left(\mathrm{M}^{+}, 44\right), 167$ (100), 139 (46), 119 (37). HRMS Found: 182.0540. Calcd. for $\mathrm{C}_{10} \mathrm{H}_{8} \mathrm{OF}_{2}: 182.0543$. Found: $\mathrm{C}, 65.78 ; \mathrm{H}, 4.35 \%$. Calcd. for $\mathrm{C}_{10} \mathrm{H}_{8} \mathrm{OF}_{2}: \mathrm{C}, 65.93 ; \mathrm{H}, 4.43 \%$.

4-(3,4,5-Trifluorophenyl)-but-3-en-2-ol (15a)-To a solution of 14a (1.08 g, $5.38 \mathrm{mmol})$ in $\mathrm{MeOH}(14 \mathrm{~mL})$ was given at $0^{\circ} \mathrm{C} \mathrm{CeCl}_{3}(1.30 \mathrm{~g}, 5.38 \mathrm{mmol})$. Then, $\mathrm{NaBH}_{4}(260 \mathrm{mg}, 6.84 \mathrm{mmol})$ was added, and the resulting solution was stirred for $5 \mathrm{~min}$. Thereafter, water $(30 \mathrm{~mL})$ was added, and the mixture was extracted with chloroform ( $3 \mathrm{X} 25 \mathrm{~mL})$. The combined organic phase was dried over anhydrous $\mathrm{MgSO}_{4}$ and concentrated in vacuo. The residue was subjected to column chromatography on silica gel (ether/ $\mathrm{CHCl} /$ hexane 1:1:1) gave 15a (939 $\mathrm{mg}, 87 \%$ ) as a colourless oil; IR (neat): $v=3364$ (bs, OH), 2976, 2926, 1618, 1530, 1440, 1043, 965, 868, $792 \mathrm{~cm}^{-1} ;{ }^{1} \mathrm{H}$ NMR $\left(270 \mathrm{MHz}, \mathrm{CDCl}_{3}\right): \delta=1.37(\mathrm{~d}$, 
$\left.3 \mathrm{H},{ }^{3} \mathrm{~J}=6.5 \mathrm{~Hz}, \mathrm{CH}_{3}\right), 1.65$ (bs, $\left.1 \mathrm{H}, \mathrm{OH}\right), 4.49\left(\mathrm{dt},{ }^{3} J=6.5 \mathrm{~Hz},{ }^{3} J=5.7 \mathrm{~Hz}\right), 6.19$ $\left(\mathrm{dd}, 1 \mathrm{H},{ }^{3} J=15.9 \mathrm{~Hz},{ }^{3} J=5.7 \mathrm{~Hz}\right), 6.44\left(\mathrm{~d}, 1 \mathrm{H},{ }^{3} J=15.9 \mathrm{~Hz}\right), 6.96\left(\mathrm{t}, 2 \mathrm{H},{ }^{3} J=7.0\right.$ $\mathrm{Hz}) \mathrm{ppm} ;{ }^{13} \mathrm{C}$ NMR $\left(67.8 \mathrm{MHz}, \mathrm{CDCl}_{3}\right): \delta=22.0,73.2,110.1(\mathrm{~m}), 127.9\left(\mathrm{dd}, J_{\mathrm{CF}}\right.$ $\left.=4.5 \mathrm{~Hz}, J_{\mathrm{CF}}=2.2 \mathrm{~Hz}\right), 132.9(\mathrm{~m}), 134.5\left(\mathrm{~d}, J_{\mathrm{CF}}=2.2 \mathrm{~Hz}\right), 139.2\left(\mathrm{~J}_{\mathrm{CF}}=-252 \mathrm{~Hz}\right.$, $\left.J_{\mathrm{CF}}=15.1 \mathrm{~Hz}\right), 151.4(\mathrm{~m}) \mathrm{ppm}$; MS (EI, $\left.70 \mathrm{eV}\right): \mathrm{m} / \mathrm{z}(\%)=202\left(\mathrm{M}^{+}, 100\right), 187$ (53), 169 (46), 159 (68), 145 (61). HRMS Found: 202.0602. Calcd. for $\mathrm{C}_{10} \mathrm{H}_{9} \mathrm{OF}_{3}$ : 202.0605 .

(E) -4-(2,4-Difluorophenyl) -but-3 -en-2-ol (15b).-14b (800 mg, $4.4 \mathrm{mmol})$, $\mathrm{CeCl}_{3}(1.30 \mathrm{~g}, 5.4 \mathrm{mmol})$ and $\mathrm{NaBH}_{4}(260 \mathrm{mg}, 6.84 \mathrm{mmol})$ in a solvent mixture of $\mathrm{MeOH}(10 \mathrm{~mL})$ and diethyl ether $(5 \mathrm{~mL})$ were reacted according to general procedure A to give $15 \mathrm{~b}(730 \mathrm{mg}, 91 \%$ ) as a colorless oil; IR (neat) $v 3348$ (bs, $\mathrm{OH}), 2972,2878,1610,1598,1502,1429,1275,1140,1089,962,851 \mathrm{~cm}^{-1} ;{ }^{1} \mathrm{H}$ NMR $\left(270 \mathrm{MHz}, \mathrm{CDCl}_{3}\right) \delta 1.38\left(\mathrm{~d}, 3 \mathrm{H},{ }^{3} J=6.5 \mathrm{~Hz}, \mathrm{CH}_{3}\right), 1.60\left(\mathrm{~d}, 1 \mathrm{H},{ }^{3} J=3.9 \mathrm{~Hz}\right.$, $\mathrm{OH}), 4.50(\mathrm{~m}, 1 \mathrm{H}), 6.28\left(\mathrm{dd}, 1 \mathrm{H},{ }^{3} J=16.2 \mathrm{~Hz},{ }^{3} J=6.5 \mathrm{~Hz}\right), 6.67\left(\mathrm{~d}, 1 \mathrm{H},{ }^{3} J=16.2\right.$ $\mathrm{Hz}), 6.76-6.87(\mathrm{~m}, 2 \mathrm{H}), 7.40(\mathrm{~m}, 1 \mathrm{H}) ;{ }^{13} \mathrm{C}-\mathrm{NMR}\left(67.8 \mathrm{MHz}, \mathrm{CDCl}_{3}\right) \delta 23.4$ $\left(\mathrm{CH}_{3}\right), 69.0(\mathrm{CH}), 104.0\left(\mathrm{dd}, \mathrm{JCF} 25.8 \mathrm{~Hz}, J_{\mathrm{CF}}=25.8 \mathrm{~Hz}\right), 111.4\left(\mathrm{dd}, J_{\mathrm{CF}}-20.7 \mathrm{~Hz}\right.$, $\left.J_{\mathrm{CF}}=2.8 \mathrm{~Hz}\right), 128.3\left(\mathrm{dd}, J_{\mathrm{CF}}=9.5 \mathrm{~Hz}, J_{\mathrm{CF}}=5.0 \mathrm{~Hz}\right), 135.8\left(\mathrm{dd}, J_{\mathrm{CF}}=4.5 \mathrm{~Hz}, J_{\mathrm{CF}}=\right.$ $1.7 \mathrm{~Hz}), 160.4\left(\mathrm{dd}, J_{\mathrm{CF}}=-233 \mathrm{~Hz}, J_{\mathrm{CF}} 12.8 \mathrm{~Hz}\right), 162.2\left(\mathrm{dd}, J_{\mathrm{CF}}=-248 \mathrm{~Hz}, J_{\mathrm{CF}}=12.3\right.$ $\mathrm{Hz}$ ); MS (EI, $70 \mathrm{eV}) \mathrm{m} / \mathrm{z}(\%) 184\left(\mathrm{M}^{+}, 82\right), 169$ (67), 127 (100). HRMS Found: 184.0701. Calcd. for $\mathrm{C}_{10} \mathrm{H}_{10} \mathrm{OF}_{2}: 184.0700$. Found: $\mathrm{C}, 64.87 ; \mathrm{H}, 5.49 \%$. Calcd. for $\mathrm{C}_{10} \mathrm{H}_{10} \mathrm{OF}_{2} .0 .1 \mathrm{H}_{2} \mathrm{O}: \mathrm{C}, 64.58 ; \mathrm{H}, 5.53 \%$.

DL-1-(4-Dodecyloxyphenyl) propan-1-ol (7a) (General Procedure for the preparation of D/L-p-(alkoxylphenyl)alkyl alcohols used in this study)-To a solution of phenol $(4,4.5 \mathrm{~g}, 47.9 \mathrm{mmol})$ and cetyltrimethylammonium bromide $(1.6 \mathrm{~g}, 5.2 \mathrm{mmol})$ in $20 \mathrm{w} \%$ aq. $\mathrm{NaOH}(480 \mathrm{~mL})$ was added dodecyl bromide $(15.2 \mathrm{~g}, 61.0 \mathrm{mmol})$, and the resulting mixture was held at $80^{\circ} \mathrm{C}$ for $5.5 \mathrm{~h}$. Thereafter water $(400 \mathrm{~mL})$ was added to the mixture, which was extracted with ether/hexane (1:1, 3 X $150 \mathrm{~mL}$ ). The organic phase was washed with water, dried over anhydrous $\mathrm{MgSO}_{4}$ and concentrated in vacuo. Column chromatography on silica gel (hexane/ether 2:1) yielded dodecyloxybenzene [17] (5a, $10 \mathrm{~g}, 80 \%$ ); IR (neat): $v=2924,2852,1600,1496,1469,1244,1171,1079,1035,796,751,690$ $\mathrm{cm}^{-1} ;{ }^{1} \mathrm{H}$ NMR $\left(270 \mathrm{MHz}, \mathrm{CDCl}_{3}\right): \delta=0.88\left(3 \mathrm{H}, \mathrm{t},{ }^{3} J=7.0 \mathrm{~Hz}\right), 1.27-1.49(18 \mathrm{H}$, m), $1.78(2 \mathrm{H}, \mathrm{m}), 3.95\left(2 \mathrm{H}, \mathrm{t},{ }^{3} \mathrm{~J}=6.7 \mathrm{~Hz}\right), 6.91(3 \mathrm{H}, \mathrm{m}), 7.27(2 \mathrm{H}, \mathrm{m}) \mathrm{ppm} ;{ }^{13} \mathrm{C}$ NMR (67.8 MHz, $\left.\mathrm{CDCl}_{3}\right): \delta=14.1,22.7,26.1,29.3,29.3,29.4,29.6,29.6,29.7$ (2C), 31.9, 67.9, 114.5 (2C), 120.4, 129.4 (2C), $159.1 \mathrm{ppm}$; MS (EI, $70 \mathrm{eV}$ ): $\mathrm{m} / z$ $(\%)=262\left(\mathrm{M}^{+}, 80\right), 94(100)$. Propionyl chloride $(4.3 \mathrm{~g}, 46.7 \mathrm{mmol})$ and after 15 min dodecyloxybenzene ( $5 \mathrm{a}, 10 \mathrm{~g}, 38.2 \mathrm{mmol}$ ) were added to a suspension of anhydrous $\mathrm{AlCl}_{3}(4.8 \mathrm{~g}, 51.6 \mathrm{mmol})$ in dichloroethane $(20 \mathrm{~mL})$ at $0^{\circ} \mathrm{C}$. The reaction mixture was stirred for $60 \mathrm{~min}$ at $0^{\circ} \mathrm{C}$ and for $30 \mathrm{~min}$. at rt. Thereafter, it was poured into a biphasic mixture of water $(100 \mathrm{~mL})$ and $\mathrm{CH}_{2} \mathrm{Cl}_{2}(150 \mathrm{~mL})$. The aqueous phase was extracted with $\mathrm{CH}_{2} \mathrm{Cl}_{2}(2 \times 60 \mathrm{~mL})$, and the organic phase was washed with water $(100 \mathrm{~mL})$, dried over anhydrous $\mathrm{MgSO}_{4}$, and concentrated in vacuo. The residue was subjected to chromatography on silica gel 
(hexane/ether/ $\mathrm{CHCl}_{3}$ 5:1:1) to give 1-(4-dodecyloxyphenyl)propan-1-one (6a, 8.5 g, 70\%); IR (KBr): $v=2920,2850,1678,1607,1511,1464,1257,1229,1179$, 1110, 1018, 955, 847, $797 \mathrm{~cm}^{-1} ;{ }^{1} \mathrm{H}$ NMR $\left(270 \mathrm{MHz}, \mathrm{CDCl}_{3}\right): \delta=0.86-1.48$ $(24 \mathrm{H}, \mathrm{m}), 1.80(2 \mathrm{H}, \mathrm{m}), 2.95\left(2 \mathrm{H}, \mathrm{q},{ }^{3} \mathrm{~J}=7.3 \mathrm{~Hz}\right), 4.01\left(2 \mathrm{H}, \mathrm{t},{ }^{3} J=6.5 \mathrm{~Hz}\right), 6.91$ $\left(2 \mathrm{H}, \mathrm{d},{ }^{3} J=8.4 \mathrm{~Hz}\right), 7.92\left(2 \mathrm{H}, \mathrm{d},{ }^{3} J=8.4 \mathrm{~Hz}\right) \mathrm{ppm} ;{ }^{13} \mathrm{C} \mathrm{NMR}\left(67.8 \mathrm{MHz}, \mathrm{CDCl}_{3}\right)$ : $\delta=8.5,14.1,22.7,26.0,29.1,29.3,29.5$ (2C), 29.6, 29.6, 30.9, 31.4, 31.9, 68.2, 114.1 (2C), 129.8, 130.2 (2C), 162.9, 199.5 ppm; MS (EI, $70 \mathrm{eV}): \mathrm{m} / z(\%)=318$ (17), 289 (100), 121 (61). To a solution of 1-(4-dodecyloxyphenyl)propan-1-one (6a, $8.5 \mathrm{~g}, 26.7 \mathrm{mmol})$ in $\mathrm{MeOH}(70 \mathrm{~mL})$ was added $\mathrm{NaBH}_{4}(1.2 \mathrm{~g}, 32 \mathrm{mmol})$ at $\mathrm{rt}$ and the resulting mixture was stirred for $30 \mathrm{~min}$ at $\mathrm{rt}$. Thereafter, the mixture was concentrated in vacuo, water $(30 \mathrm{~mL})$ was added and the mixture was extracted with ether $(2 \mathrm{X} 30 \mathrm{~mL})$ and chloroform $(30 \mathrm{~mL})$. The combined organic phase was dried over anhydrous $\mathrm{MgSO}_{4}$, concentrated in vacuo, and the residue was subjected to column chromatography on silica gel (hexane/ether/ $\mathrm{CHCl}_{3}$ 1:1:1) to give DL-(4-dodecyloxyphenyl)propyl alcohol (7a) as a colourless solid (8.0 g, 93\%); M.p.: $38^{\circ} \mathrm{C}$; IR (KBr): $v=3402$ (bs, OH), 2920, 2848, 1612, 1512, $1468,1246,1175,1027,836,813,722 \mathrm{~cm}^{-1} ;{ }^{1} \mathrm{H} \mathrm{NMR}\left(270 \mathrm{MHz}, \mathrm{CDCl}_{3}\right): \delta=0.89$ $(6 \mathrm{H}, \mathrm{m}), 1.27-1.83(23 \mathrm{H}, \mathrm{m}), 3.94\left(2 \mathrm{H}, \mathrm{t},{ }^{3} J=6.7 \mathrm{~Hz}\right), 4.53(1 \mathrm{H}, \mathrm{m}), 6.86(2 \mathrm{H}, \mathrm{d}$, $\left.{ }^{3} J=8.4 \mathrm{~Hz}\right), 7.25\left(2 \mathrm{H}, \mathrm{d},{ }^{3} J=8.4 \mathrm{~Hz}\right) \mathrm{ppm} ;{ }^{13} \mathrm{C} \mathrm{NMR}\left(67.8 \mathrm{MHz}, \mathrm{CDCl}_{3}\right): \delta=$ 10.2, 14.1, 22.7, 26.1, 29.3, 29.4, 29.4, 29.6, 29.6, 29.6, 29.7, 31.8, 31.9, 68.1, 75.7, 114.4 (2C), 127.2 (2C), 136.5, 158.6 ppm; MS (FAB, 3-nitrobenzyl alcohol): m/z $(\%)=320\left(\mathrm{M}^{+}\right), 303\left(\mathrm{MH}^{+}-\mathrm{H}_{2} \mathrm{O}, 100\right), 291$ (62), 135 (44) 123 (31). HRMS Found: 320.2712. Calcd. for $\mathrm{C}_{21} \mathrm{H}_{36} \mathrm{O}_{2}: 320.2715$.

\section{Liquid Etherification of benzyl alcohols with $\mathrm{Pt} / \mathrm{C}$ and $\mathrm{Pd} / \mathrm{C}$}

Bis(4-methoxybenzyl) ether (16a) [24]-A mixture of 4-methoxybenzyl alcohol (3e, $6.0 \mathrm{~g}, 43.4 \mathrm{mmol})$ and Pt/C (50 mg, $5 \mathrm{wt} \% \mathrm{Pt}, 0.012 \mathrm{mmol} \mathrm{Pt}, 0.03$ mol\% Pt, Kishida) was kept at $135^{\circ} \mathrm{C}$ for $15 \mathrm{~h}$. Thereafter, the cooled mixture was filtered over a short column of florisil (hexane) to give, after evaporation of the hexane, bis(4-methoxybenzyl) ether (16a) (5.3 g, 95\%) as a slowly crystallizing, colorless oil, M.p. $37^{\circ} \mathrm{C}$; IR (neat) $v=2922,1611,1512,1478,1451,1247,1179$, $1048,922,826 \mathrm{~cm}^{-1} ;{ }^{1} \mathrm{H}$ NMR $\left(270 \mathrm{MHz} \mathrm{CDCl}_{3}\right): \delta=3.80\left(6 \mathrm{H}, \mathrm{s}, 2 \mathrm{OCH}_{3}\right), 4.45$ $\left(4 \mathrm{H}, \mathrm{s}, 2 \mathrm{OCH}_{2}\right), 6.88\left(4 \mathrm{H}, \mathrm{d},{ }^{3} J=8.9 \mathrm{~Hz}\right), 7.28\left(4 \mathrm{H}, \mathrm{d},{ }^{3} J=8.9 \mathrm{~Hz}\right) \mathrm{ppm} ;{ }^{13} \mathrm{C}$ NMR (67.8 MHz, $\mathrm{CDCl}_{3}$ ): $\delta=55.3$ (2C), 71.5 (2C), 113.8 (4C), 129.4 (4C), 130.5 (2C), 159.2 (2C) ppm; MS (EI, $70 \mathrm{eV}): m / z(\%)=258\left(\mathrm{M}^{+}, 29\right), 150(17), 121$ (100).

Bis(4-pentoxybenzyl) ether(16d)-A mixture of 4-pentoxybenzyl alcohol (3d, $1.94 \mathrm{~g}, 10.0 \mathrm{mmol}$ ) and Pt/C (50 mg, $5 \mathrm{wt} \% \mathrm{Pt}, 0.012 \mathrm{mmol} \mathrm{Pt}, 0.12 \mathrm{~mol} \%$ $\mathrm{Pt}$, Kishida) was kept at $135^{\circ} \mathrm{C}$ for $15 \mathrm{~h}$. Thereafter, the crude mixture was subjected to column chromatography on silica gel (hexane/ether/ $\mathrm{CHCl}_{3}$ 7:1:1) to give $16 \mathrm{~d}(1.42 \mathrm{~g}, 77 \%)$ as a slowly crystallizing, colorless oil; IR (neat) $v=2930$, 2858, 1613, 1513, 1471, 1243, 1171, 1076, 1026, $826 \mathrm{~cm}^{-1} ;{ }^{1} \mathrm{H}$ NMR $(270 \mathrm{MHz}$, $\left.\mathrm{CDCl}_{3}\right): \delta=0.93\left(6 \mathrm{H}, \mathrm{t},{ }^{3} J=7.0 \mathrm{~Hz}\right), 1.44(8 \mathrm{H}, \mathrm{m}), 1.75(4 \mathrm{H}, \mathrm{m}), 3.95\left(4 \mathrm{H}, \mathrm{t},{ }^{3} J=\right.$ $6.5 \mathrm{~Hz}), 4.45(4 \mathrm{H}, \mathrm{s}), 6.87\left(4 \mathrm{H}, \mathrm{d},{ }^{3} J=8.4 \mathrm{~Hz}\right) ; 7.25\left(4 \mathrm{H}, \mathrm{d},{ }^{3} J=8.4 \mathrm{~Hz}\right) \mathrm{ppm} ;{ }^{13} \mathrm{C}$ 
NMR (67.8 MHz, $\mathrm{CDCl}_{3}$ ): $\delta=14.1$ (2C), 22.5 (2C), 28.2 (2C), 29.0 (2C), 68.0 (2C), 71.6 (2C), 114.4 (4C), 129.4 (4C), 130.3 (2C), 158.9 (2C) ppm; MS (EI, 70 $\mathrm{eV}): m / z(\%)=370\left(\mathrm{M}^{+}, 20\right), 206(28), 178$ (37), 107 (100). HRMS Found: 370.2508. Calcd. for $\mathrm{C}_{24} \mathrm{H}_{34} \mathrm{O}_{3}: 370.2508$.

Further bisbenzyl ethers were produced according to procedure A:

Bis(4-ethoxybenzyI) ether(16b) [24], colorless, slowly crystallizing oil, IR (neat) $v=2930,1244 \mathrm{~cm}^{-1} ;{ }^{1} \mathrm{H} \mathrm{NMR}\left(270 \mathrm{MHz}, \mathrm{CDCl}_{3}\right): \delta=1.37\left(6 \mathrm{H}, \mathrm{t},{ }^{3} J=\mathrm{Hz}\right)$, $3.96\left(4 \mathrm{H}, \mathrm{q},{ }^{3} J=\mathrm{Hz}\right), 4.45(4 \mathrm{H}, \mathrm{s}), 6.87\left(4 \mathrm{H}, \mathrm{d},{ }^{3} J=8.4 \mathrm{~Hz}\right) ; 7.25\left(4 \mathrm{H}, \mathrm{d},{ }^{3} J=8.4\right.$ $\mathrm{Hz}) \mathrm{ppm}$; MS (EI, $70 \mathrm{eV}): \mathrm{m} / \mathrm{z}(\%)=286\left(\mathrm{M}^{+}, 23\right)$.

Bis(4-propoxybenzyl) ether (16c), colorless, slowly crystallizing oil, IR (neat) $v=2928,1245 \mathrm{~cm}^{-1} ;{ }^{1} \mathrm{H} \mathrm{NMR}\left(270 \mathrm{MHz}, \mathrm{CDCl}_{3}\right): \delta=1.08\left(6 \mathrm{H}, \mathrm{t},{ }^{3} J=6.9 \mathrm{~Hz}\right)$, $1.82\left(4 \mathrm{H}, \mathrm{dd},{ }^{3} J=6.9 \mathrm{~Hz},{ }^{3} J=6.6 \mathrm{~Hz}\right), 3.92\left(\mathrm{~d}, 4 \mathrm{H},{ }^{3} J=6.6 \mathrm{~Hz}\right), 4.44(4 \mathrm{H}, \mathrm{s}), 6.87$ $\left(4 \mathrm{H}, \mathrm{d},{ }^{3} J=8.5 \mathrm{~Hz}\right), 7.27\left(4 \mathrm{H}, \mathrm{d},{ }^{3} J=8.5 \mathrm{~Hz}\right) \mathrm{ppm}$; MS (EI, $\left.70 \mathrm{eV}\right): \mathrm{m} / z(\%)=$ $314\left(\mathrm{M}^{+}, 13\right)$.

Bis(3,4-dimethoxybenzy) ether (16e) [25], colorless solid, m.p. $73^{\circ} \mathrm{C}$, IR $(\mathrm{KBr}) v=3000,2937,1260,1152,1026 \mathrm{~cm}^{-1} ;{ }^{1} \mathrm{H} \mathrm{NMR}\left(270 \mathrm{MHz}, \mathrm{CDCl}_{3}\right): \delta=$ $3.88\left(6 \mathrm{H}, \mathrm{s}, 2 \mathrm{OCH}_{3}\right), 3.89\left(6 \mathrm{H}, \mathrm{s}, 2 \mathrm{OCH}_{3}\right), 4.46(4 \mathrm{H}, \mathrm{s}), 6.88-6.94(6 \mathrm{H}, \mathrm{m})$ ppm; MS (EI, $70 \mathrm{eV}): m / z(\%)=318\left(\mathrm{M}^{+}\right)(23), 152(100)$.

Bis(4-methylbenzyl) ether (16f) [26]-colorless solid; m.p. 61 ${ }^{\circ} \mathrm{C}$ [27] IR (KBr) $v=3066,2960,2870,1450,1100 ;{ }^{1} \mathrm{H} \mathrm{NMR}\left(270 \mathrm{MHz}, \mathrm{CDCl}_{3}\right): \delta=2.37(6 \mathrm{H}, \mathrm{s}, 2$ $\left.\mathrm{CH}_{3}\right), 4.54\left(4 \mathrm{H}, \mathrm{s}, 2 \mathrm{OCH}_{2}\right), 7.20\left(4 \mathrm{H}, \mathrm{d},{ }^{3} J=7.6 \mathrm{~Hz}\right), 7.29\left(4 \mathrm{H}, \mathrm{d},{ }^{3} J=7.6 \mathrm{~Hz}\right)$; ${ }^{13} \mathrm{C}$ NMR (67.8 MHz, $\mathrm{CDCl}_{3}$, DEPT 90, DEPT 135): $\delta=21.4\left(2 \mathrm{C}, 2 \mathrm{CH}_{3}\right), 71.9$ $\left(2 \mathrm{C}, 2 \mathrm{OCH}_{2}\right), 128.1(4 \mathrm{C}, 4 \mathrm{CH}), 129.4(4 \mathrm{C}, \mathrm{CH}), 135.7\left(2 \mathrm{C}, \mathrm{C}_{\text {quat }}\right), 137.8(2 \mathrm{C}$, $\left.\mathrm{C}_{\text {quat }}\right)$; MS $(70 \mathrm{eV}) \mathrm{m} / \mathrm{z}(\%)=226\left(\mathrm{M}^{+}\right)(18)$.

Oxidation of benzhydrols 17 to benzophenones 18 with $20 \mathrm{w} \% \mathrm{Pt}-\mathrm{Ru}$ on CNF

Benzophenone(18a) - A mixture of benzhydrol (17a, $150 \mathrm{mg}, 0.82 \mathrm{mmol})$ and $20 \mathrm{wt} \% \mathrm{Pt}-\mathrm{Ru}$ on CNF [17] (25 mg) in well-aerated diphenyl ether (700 mg) was heated to $135^{\circ} \mathrm{C}$ for $3 \mathrm{~h}$. Thereafter, the cooled reaction mixture was subjected directly to column chromatography (hexane, to elute diphenyl ether, then hexane/ether 3:1) to give benzophenone (130 mg, $88 \%)$, mp. $47^{\circ} \mathrm{C}$; IR $(\mathrm{KBr}) v=$ 1670, 1450, 1280, 705, $690 \mathrm{~cm}^{-1} ;{ }^{1} \mathrm{H}$ NMR $\left(270 \mathrm{MHz}, \mathrm{CDCl}_{3}\right): 7.45-7.58(6 \mathrm{H}$, m), 7.79 - $7.83(4 \mathrm{H}, \mathrm{m}) ;{ }^{13} \mathrm{C} \mathrm{NMR}\left(67.8 \mathrm{MHz}, \mathrm{CDCl}_{3}\right.$, DEPT 90, DEPT 135): $\delta=$ $128.2(4 \mathrm{C}, \mathrm{CH}), 130.0(4 \mathrm{C}, \mathrm{CH}), 132.3(2 \mathrm{C}, \mathrm{CH}), 137.6\left(2 \mathrm{C}, \mathrm{C}_{\text {quat }}\right), 196.5\left(\mathrm{C}_{\text {quat }}\right.$, $\mathrm{CO})$; MS (EI, $70 \mathrm{eV}): \mathrm{m} / z$ (\%) $182\left(\mathrm{M}^{+}\right)$(15), 105 (100).

Analogously was prepared: 4,4'-dimethylbenzophenone (18b) [28] as a colorless solid, mp. $91^{\circ} \mathrm{C}$; IR (KBr) $v=1645,1610,1315,1280,750 \mathrm{~cm}^{-1}$; ${ }^{1} \mathrm{H}$ NMR $\left(270 \mathrm{MHz}, \mathrm{CDCl}_{3}\right): 2.44\left(6 \mathrm{H}, \mathrm{s}, 2 \mathrm{CH}_{3}\right), 7.26\left(4 \mathrm{H}, \mathrm{d},{ }^{3} J=8.0 \mathrm{~Hz}\right), 7.70\left(4 \mathrm{H}, \mathrm{d},{ }^{3} J=\right.$ $8.0 \mathrm{~Hz}) ;{ }^{13} \mathrm{C}$ NMR (67.8 MHz, $\mathrm{CDCl}_{3}$, DEPT 90, DEPT 135): $129.1(4 \mathrm{C}, \mathrm{CH})$, $130.1(4 \mathrm{C}, \mathrm{CH}), 135.4\left(2 \mathrm{C}, \mathrm{C}_{\text {quat }}\right), 143.0\left(\mathrm{C}_{\text {quat }}\right), 196.1\left(\mathrm{C}_{\text {quat, }} \mathrm{CO}\right)$; $\mathrm{MS}(70 \mathrm{eV}) \mathrm{m} / \mathrm{z}$ (\%) $210\left(\mathrm{M}^{+}\right)(23), 119(100)$.

Liquid Etherification of 1 -phenylethanols, phenylpropanols and cinnamyl alcohols with $P t / C$ and $P d / C$ 
Bis-1-(4-methoxypheny) ethyl ether (19a)-A mixture of 9a (1.56 g, 10.3 $\mathrm{mmol}$ ) and $\mathrm{Pd} / \mathrm{C}(30 \mathrm{mg}, 10 \mathrm{w} \% \mathrm{Pd}, 0.027 \mathrm{mmolPd}, 0.026 \mathrm{~mol} \% \mathrm{Pd}$, Wako) was kept at $135^{\circ} \mathrm{C}$ for $30 \mathrm{~min}$. Thereafter, the cooled residue was subjected to rapid chromatography on silica gel (hexane.ether/ $\left.\mathrm{CHCl}_{3} 5: 1: 1\right)$ to give $19 \mathrm{a}(1.27 \mathrm{~g}$, $86 \%$ ) as a colorless oil; IR (neat, for A/B): $v=2968,2832,1612,1512,1300,1246$, $1173,1090,1037,830,810 \mathrm{~cm}^{-1}$; ${ }^{1} \mathrm{H}$ NMR $\left(270 \mathrm{MHz}, \mathrm{CDCl}_{3}\right): \delta=1.33$ (B, $3 \mathrm{H}, \mathrm{d}$, $\left.{ }^{3} J=6.5 \mathrm{~Hz}, \mathrm{CH}_{3}\right), 1.41\left(\mathrm{~A}, 3 \mathrm{H}, \mathrm{d},{ }^{3} J=6.5 \mathrm{~Hz}, \mathrm{CH}_{3}\right), 3.77\left(\mathrm{~A}, 3 \mathrm{H}, \mathrm{s}, \mathrm{OCH}_{3}\right), 3.80$ $\left(\mathrm{B}, 3 \mathrm{H}, \mathrm{s}, \mathrm{OCH}_{3}\right), 4.16\left(\mathrm{~B}, 1 \mathrm{H}, \mathrm{q}, \mathrm{OCH},{ }^{3} J=6.5 \mathrm{~Hz}\right), 4.42\left(\mathrm{~A}, 1 \mathrm{H}, \mathrm{q}, \mathrm{OCH},{ }^{3} J=6.5\right.$ $\mathrm{Hz}), 6.81\left(\mathrm{~A}, 2 \mathrm{H}, \mathrm{d},{ }^{3} J=8.4 \mathrm{~Hz}\right), 6.87\left(\mathrm{~B}, \mathrm{~d}, 2 \mathrm{H},{ }^{3} J=8.4 \mathrm{~Hz}\right), 7.17\left(\mathrm{~A} / \mathrm{B}, 2 \mathrm{H}, \mathrm{d},{ }^{3} J\right.$ $=8.4 \mathrm{~Hz}) \mathrm{ppm} ;{ }^{13} \mathrm{C}$ NMR $\left(67.8 \mathrm{MHz}, \mathrm{CDCl}_{3}\right): \delta=22.8\left(\mathrm{~A},+, \mathrm{CH}_{3}\right), 24.7(\mathrm{~B},+$, $\left.\mathrm{CH}_{3}\right), 55.2\left(\mathrm{~A} / \mathrm{B},+, \mathrm{OCH}_{3}\right), 73.7(\mathrm{~A},+, \mathrm{OCH}), 73.8(\mathrm{~B},+, \mathrm{OCH}), 113.6(\mathrm{~A}, 2 \mathrm{C},+$, $\mathrm{CH}), 113.8(\mathrm{~B}, 2 \mathrm{C},+, \mathrm{CH}), 127.4(\mathrm{~A}, 2 \mathrm{C},+, \mathrm{CH}), 127.5(\mathrm{~B}, 2 \mathrm{C},+, \mathrm{CH}), 136.2(\mathrm{~B}$, $\left.\mathrm{C}_{\text {quat }}\right), 136.6\left(\mathrm{~A}, \mathrm{C}_{\text {quat }}\right), 158.7\left(\mathrm{~A}, \mathrm{C}_{\text {quat }}\right), 158.9\left(\mathrm{~B}, \mathrm{C}_{\text {quat }}\right) \mathrm{ppm}$; MS $(70 \mathrm{eV}): \mathrm{m} / z(\%)$ for $\mathrm{A} / \mathrm{B}=286\left(\mathrm{M}^{+}, 28\right), 178(18), 135$ (100). HRMS Found: 286.1568. Calcd. for $\mathrm{C}_{18} \mathrm{H}_{22} \mathrm{O}_{3}: 286.1569$

(D/L)-Bis-(4-[3,4,5-trifluorophenyl]but-3-en-2-yl)ether(22a)-A mixture of $15 \mathrm{a}(2.08 \mathrm{~g}, 10.3 \mathrm{mmol})$ and $\mathrm{Pd} / \mathrm{C}(30 \mathrm{mg}, 10 \mathrm{w} \% \mathrm{Pd}, 0.027 \mathrm{mmolPd}, 0.026$ mol\% Pd, Wako) was kept at $135^{\circ} \mathrm{C}$ for $45 \mathrm{~min}$. Thereafter, the cooled residue was subjected to rapid chromatography on silica gel (hexane/ether $/ \mathrm{CHCl}_{3}$ 8:1:1) to give (D/L-22a) $(680 \mathrm{mg}, 34 \%)$ as a colourless solid, M.p. $89^{\circ} \mathrm{C}$; IR $(\mathrm{KBr}): v=$ 3076, 2970, 2930, 2888, 1618, 1532, 1438, 1336, 1230, 1140, 1072, 1041, 966, 870, $836,825,790,663,618,424 \mathrm{~cm}^{-1} ;{ }^{1} \mathrm{H}$ NMR $\left(270 \mathrm{MHz}, \mathrm{CDCl}_{3}\right): \delta=1.31\left(6 \mathrm{H}, \mathrm{d},{ }^{3} J\right.$ $\left.=6.5 \mathrm{~Hz}, 2 \mathrm{CH}_{3}\right), 4.09\left(2 \mathrm{H}, \mathrm{dt},{ }^{3} J=7.3 \mathrm{~Hz},{ }^{3} J=6.5 \mathrm{~Hz}\right), 6.06\left(2 \mathrm{H}, \mathrm{dd},{ }^{3} J=16.2\right.$ $\left.\mathrm{Hz},{ }^{3} J=7.3 \mathrm{~Hz}\right), 6.35\left(2 \mathrm{H}, \mathrm{d},{ }^{3} J=16.2 \mathrm{~Hz}\right), 6.98(4 \mathrm{H}, \mathrm{m}) \mathrm{ppm}$; MS (EI, $\left.70 \mathrm{eV}\right)$ : $m / z(\%)=386\left(\mathrm{M}^{+}, 0.4\right), 342(67), 185$ (100), 145 (71). HRMS Found: 386.1107. Calcd. for $\mathrm{C}_{20} \mathrm{H}_{16} \mathrm{OF}_{6}: 386.1105$; Found: $\mathrm{C}, 62.14 ; \mathrm{H}, 4.18 \%$. Calcd. for $\mathrm{C}_{20} \mathrm{H}_{16} \mathrm{~F}_{6} \mathrm{O}$ : $\mathrm{C}, 62.18 ; \mathrm{H}, 4.17 \%$; and meso-bis-(4-[3,4,5-trifluorophenyl]but-3-en-2-yl)ether (meso-22a, $\left.\mathrm{C}_{20} \mathrm{H}_{16} \mathrm{OF}_{6}\right)(640 \mathrm{mg}, 32 \%)$ as a colourless oil; IR (neat): $v=2974$, 2926, 2868, 1616, 1529, 1439, 1359, 1305, 1256, 1232, 1144, 1042, 964, 869, 792, $702,619 \mathrm{~cm}^{-1} ;{ }^{1} \mathrm{H}$ NMR $\left(270 \mathrm{MHz}, \mathrm{CDCl}_{3}\right): \delta=1.33\left(6 \mathrm{H}, \mathrm{d},{ }^{3} J=6.5 \mathrm{~Hz}, 2 \mathrm{CH}_{3}\right)$, $4.17\left(2 \mathrm{H}, \mathrm{dt},{ }^{3} J=6.7 \mathrm{~Hz},{ }^{3} J=6.5 \mathrm{~Hz}\right), 6.08\left(2 \mathrm{H}, \mathrm{dd},{ }^{3} J=15.9 \mathrm{~Hz},{ }^{3} J=6.7 \mathrm{~Hz}\right)$, $6.37\left(2 \mathrm{H}, \mathrm{d},{ }^{3} J=15.9 \mathrm{~Hz}\right), 6.93(4 \mathrm{H}, \mathrm{m}) \mathrm{ppm}$; MS (EI, $\left.70 \mathrm{eV}\right): \mathrm{m} / z(\%)=386\left(\mathrm{M}^{+}\right.$, 30), 342 (100), 185 (32), 145 (17). HRMS Found: 386.1107. Calcd. for $\mathrm{C}_{20} \mathrm{H}_{16} \mathrm{OF}_{6}$ : 386.1105 .

Reaction of (E)-4-(2,4-difluorophenyl)but-3-en-2-ol(15b) over $\mathrm{Pd} / \mathrm{C}-15 \mathrm{~b}$ $(1.20 \mathrm{~g}, 6.5 \mathrm{mmol})$ was heated over $\mathrm{Pd} / \mathrm{C}(30 \mathrm{mg})$ at $135^{\circ} \mathrm{C}$ for $1 \mathrm{~h}$. The reaction mixture was subjected directly to column chromatography on silica gel to give (eluted with hexane) (E)-1-(2,4-difluorophenyl)but-1-ene (23b) (145 mg, 13\%) as a colorless oil; IR (neat) $v 3040,2964,2928,2874,1617,1500,1459,1428$, $1273,1140,1087,966,851,803,607,591 \mathrm{~cm}^{-1} ;{ }^{1} \mathrm{H}$ NMR $\left(270 \mathrm{MHz}, \mathrm{CDCl}_{3}\right) \delta 1.10$ $\left(\mathrm{t}, 3 \mathrm{H},{ }^{3} \mathrm{~J}=7.3 \mathrm{~Hz}\right), 2.24\left(\mathrm{dt}, 2 \mathrm{H},{ }^{3} J=7.3 \mathrm{~Hz},{ }^{3} J=6.2 \mathrm{~Hz}\right), 6.26\left(\mathrm{dt}, 1 \mathrm{H},{ }^{3} J=15.9\right.$ $\left.\mathrm{Hz},{ }^{3} J=6.2 \mathrm{~Hz}\right), 6.48\left(\mathrm{~d}, 1 \mathrm{H},{ }^{3} J=15.9 \mathrm{~Hz}\right), 6.73-6.83(\mathrm{~m}, 2 \mathrm{H}), 7.39(\mathrm{~m}, 1 \mathrm{H})$; ${ }^{13} \mathrm{C}-\mathrm{NMR}\left(67.8 \mathrm{MHz}, \mathrm{CDCl}_{3}\right) \delta 13.5\left(\mathrm{CH}_{3}\right), 26.4\left(\mathrm{CH}_{2}\right), 103.8\left(\mathrm{dd}, J_{\mathrm{CF}}=26.2 \mathrm{~Hz}\right.$ 
$\left.J_{\mathrm{CF}}=26.2 \mathrm{~Hz}\right), 111.2\left(\mathrm{dd}, J_{\mathrm{CF}}=21.2 \mathrm{~Hz}, J_{\mathrm{CF}}=3.9 \mathrm{~Hz}\right), 120.3\left(\mathrm{dd}, J_{\mathrm{CF}}=2.8 \mathrm{~Hz}, J_{\mathrm{CF}}\right.$ $=1.2 \mathrm{~Hz}), 122.0\left(\mathrm{dd}, J_{\mathrm{CF}}=12.3 \mathrm{~Hz}, J_{\mathrm{CF}}=3.9 \mathrm{~Hz}\right), 127.6\left(\mathrm{dd}, J_{\mathrm{CF}}=8.9 \mathrm{~Hz}, J_{\mathrm{CF}}=5.6\right.$ $\mathrm{Hz}), 134.9\left(\mathrm{dd}, J_{\mathrm{CF}}=3.9 \mathrm{~Hz}, J_{\mathrm{CF}}=2.2 \mathrm{~Hz}\right), 159.7\left(\mathrm{dd}, J_{\mathrm{CF}}=-250 \mathrm{~Hz}, J_{\mathrm{CF}}=11.8\right.$ $\mathrm{Hz}), 161.6\left(\mathrm{dd}, J_{\mathrm{CF}}=-248 \mathrm{~Hz}, J_{\mathrm{CF}}=11.7 \mathrm{~Hz}\right)$; MS (EI, $\left.70 \mathrm{eV}\right) \mathrm{m} / z(\%) 168\left(\mathrm{M}^{+}\right.$, 57), 153 (100), 127 (45); HRMS Found: 168.0753. Calcd. for $\mathrm{C}_{10} \mathrm{H}_{10} \mathrm{~F}_{2}: 168.0751$ and (eluted with hexane) (E)-1-(2,4-difluorophenyl)buta-1,3-diene (23a) (76 $\mathrm{mg}, 7 \%$ ) as a colorless oil; IR (neat) $v 2924,1615,1504,1431,1276,1140,1088$, $1004,966,907,852,807,729 \mathrm{~cm}^{-1} ;{ }^{1} \mathrm{H}$ NMR $\left(270 \mathrm{MHz}, \mathrm{CDCl}_{3}\right) \delta 5.21(\mathrm{~d}, 1 \mathrm{H}, J=$ $10.0 \mathrm{~Hz}), 5.36(\mathrm{~d}, 1 \mathrm{H}, J=17.0 \mathrm{~Hz}), 6.53(\mathrm{~m}, 1 \mathrm{H}), 6.70(\mathrm{~m}, 1 \mathrm{H}), 6.74-6.87(\mathrm{~m}$, $3 \mathrm{H}), 7.44(\mathrm{~m}, 1 \mathrm{H}) ;{ }^{13} \mathrm{C}-\mathrm{NMR}\left(67.8 \mathrm{MHz}, \mathrm{CDCl}_{3}\right) \delta 104.1\left(\mathrm{dd}, J_{\mathrm{CF}}=25.8 \mathrm{~Hz}, J_{\mathrm{CF}}=\right.$ $25.8 \mathrm{~Hz}), 111.5\left(\mathrm{dd}, J_{\mathrm{CF}}=21.8 \mathrm{~Hz}, \mathrm{JCF}=3.9 \mathrm{~Hz}\right), 118.5,121.4\left(\mathrm{dd}, J_{\mathrm{CF}}=12.3 \mathrm{~Hz}\right.$, $\left.J_{\mathrm{CF}} 3.9 \mathrm{~Hz}\right), 124.1\left(\mathrm{dd}, J_{\mathrm{CF}}=2.8 \mathrm{~Hz}, J_{\mathrm{CF}}=2.8 \mathrm{~Hz}\right), 127.9\left(\mathrm{dd}, J_{\mathrm{CF}}=9.5 \mathrm{~Hz}, J_{\mathrm{CF}}=5.0\right.$ $\mathrm{Hz}), 131.6\left(\mathrm{dd}, J_{\mathrm{CF}}=5.0 \mathrm{~Hz}, J_{\mathrm{CF}}=2.8 \mathrm{~Hz}\right), 137.2,160.3\left(\mathrm{dd}, J_{\mathrm{CF}}=-252 \mathrm{~Hz}, J_{\mathrm{CF}}=\right.$ $11.7 \mathrm{~Hz}), 162.2\left(\mathrm{dd}, J_{\mathrm{CF}}=-249 \mathrm{~Hz}, J_{\mathrm{CF}}=11.7 \mathrm{~Hz}\right) ; \mathrm{MS}(\mathrm{EI}, 70 \mathrm{eV}) \mathrm{m} / \mathrm{z}(\%) 166$ $\left(\mathrm{M}^{+}, 22\right), 151$ (12), 127 (9). HRMS Found: 166.0591. Calcd. for $\mathrm{C}_{10} \mathrm{H}_{8} \mathrm{~F}_{2}$ : 166.0594, and (eluted with hexane/ $\left.\mathrm{Et}_{2} \mathrm{O} / \mathrm{CHCl}_{3}\right)(D / L)$ - and meso-bis(4-[2,4difluorophenyl]but-3-en-2-yl)ether (22b, $385 \mathrm{mg}, 37 \%$ [15]). Further elution with hexane/ $\mathrm{Et}_{2} \mathrm{O} / \mathrm{CHCl}_{3}$ provided (E)-4-(2,4-difluorophenyl)but-3-en-2-one (23c, $178 \mathrm{mg}, 15 \%), 2,4$-difluorophenylbutan-2-one (23d, $190 \mathrm{mg}, 16 \%$ [15]) and 4-(2,4-difluorophenyl)butan-2-ol (23e, $180 \mathrm{mg}, 15 \%)$ as a colorless oil; IR (neat) v 3384 (bs, OH), 2924, 1618, 1501, 1459, 1424, 1375, 1279, 1138, 1101, $968,847,813,728 \mathrm{~cm}^{-1}$; ${ }^{1} \mathrm{H}$ NMR $\left(270 \mathrm{MHz}, \mathrm{CDCl}_{3}\right) \delta 1.23\left(\mathrm{~d}, 3 \mathrm{H},{ }^{3} J=6.2 \mathrm{~Hz}\right.$, $\left.\mathrm{CH}_{3}\right), 1.56$ (bs, $\left.1 \mathrm{H}, \mathrm{OH}\right), 1.73(\mathrm{~m}, 2 \mathrm{H}), 2.71(\mathrm{~m}, 2 \mathrm{H}), 3.81(\mathrm{~m}, 1 \mathrm{H}), 6.78(\mathrm{~m}, 2 \mathrm{H})$, $7.15(\mathrm{~m}, 1 \mathrm{H}) ;{ }^{13} \mathrm{C}-\mathrm{NMR}\left(67.8 \mathrm{MHz}, \mathrm{CDCl}_{3}\right) \delta 23.6\left(\mathrm{CH}_{3}\right), 24.8\left(\mathrm{CH}_{2}\right), 39.4\left(\mathrm{CH}_{2}\right)$, $67.3(\mathrm{CH}), 103.6\left(\mathrm{dd}, J_{\mathrm{CF}}=26.2 \mathrm{~Hz}, J_{\mathrm{CF}}=25.1 \mathrm{~Hz}\right), 110.9\left(\mathrm{dd}, J_{\mathrm{CF}}=20.7 \mathrm{~Hz}, J_{\mathrm{CF}}=\right.$ $3.3 \mathrm{~Hz}), 124.6\left(\mathrm{dd}, J_{\mathrm{CF}}=16.2 \mathrm{~Hz}, J_{\mathrm{CF}}=3.9 \mathrm{~Hz}\right), 131.0\left(\mathrm{dd}, J_{\mathrm{CF}}=9.5 \mathrm{~Hz}, \mathrm{~J}_{\mathrm{CF}}-7.3\right.$ $\mathrm{Hz}), 160.9\left(\mathrm{dd}, J_{\mathrm{CF}}=-246 \mathrm{~Hz}, J_{\mathrm{CF}}=11.8 \mathrm{~Hz}\right), 161.4\left(\mathrm{dd}, J_{\mathrm{CF}}=-246 \mathrm{~Hz}, J_{\mathrm{CF}}=12.3\right.$ $\mathrm{Hz}$ ); MS (EI, $70 \mathrm{eV}$ ) m/z (\%) 186 (M+2, 2.2), 168 (57), 153 (66), 127 (73). HRMS Found: 168.0854. Calcd. for $\mathrm{C}_{10} \mathrm{H}_{12} \mathrm{OF}_{2}: 186.0856$, and starting material $15 \mathrm{~b}$.

\section{Analogous reactions yielded:}

DL- and meso-Bis-1-(4-methylphenyl)ethyl ether (19b); colorless oil; IR $\left(\right.$ neat $\left./ \mathrm{cm}^{-1}\right): v=2972,2929,2864,1512,1445,1367,1302,1092,1024,947,816$ $\mathrm{cm}^{-1} ;{ }^{1} \mathrm{H} \mathrm{NMR}\left(270 \mathrm{MHz}, \mathrm{CDCl}_{3}\right): \delta=1.35\left(3 \mathrm{H}, \mathrm{d},{ }^{3} J=6.5 \mathrm{~Hz}, \mathrm{CH}_{3}\right), 1.43(3 \mathrm{H}, \mathrm{d}$, $\left.{ }^{3} J=6.5 \mathrm{~Hz}, \mathrm{CH}_{3}\right), 2.32\left(3 \mathrm{H}, \mathrm{s}, \mathrm{CH}_{3}\right), 2.36\left(3 \mathrm{H}, \mathrm{s}, \mathrm{CH}_{3}\right), 4.21\left(\mathrm{q}, 1 \mathrm{H}, \mathrm{OCH},{ }^{3} J=6.8\right.$ $\mathrm{Hz}), 4.49\left(\mathrm{q}, 1 \mathrm{H}, \mathrm{OCH},{ }^{3} J=6.8 \mathrm{~Hz}\right), 7.06-7.29(4 \mathrm{H}, \mathrm{m}, \mathrm{A} / \mathrm{B}) \mathrm{ppm} ;{ }^{13} \mathrm{C} \mathrm{NMR}$ (67.8 MHz, $\left.\mathrm{CDCl}_{3}\right): \delta=21.0$ [8], (21.1 [4]), 22.8 [6], (24.6 [9]), 73.9 [2], (74.2 [4]), 126.1 [8], (126.2 [8]), 129.1 [0], (129.1 [9]), 136.6 [7], (136.9 [5]), (141.1 [6]), 141.3 [2] ppm; MS (EI, $70 \mathrm{eV}): \mathrm{m} / \mathrm{z}(\%)=254\left(\mathrm{M}^{+}, 100\right), 239\left(\mathrm{M}^{+}-\mathrm{CH}_{3}, 88\right)$, 153 (27), 136 (19), 119 (52). HRMS Found: 254.1668. Calcd. for $\mathrm{C}_{18} \mathrm{H}_{22} \mathrm{O}$ : 254.1671.

Bis-1-(4-methoxypheny) propyl ether (19c); slowly crystallizing oil (Lit. mp. $\left.64^{\circ} \mathrm{C}\right)$; IR (neat, for A/B): $v 2968,2832,1611,1253,1040 \mathrm{~cm}^{-1} ;{ }^{1} \mathrm{H}$ NMR $(270$ $\left.\mathrm{MHz}, \mathrm{CDCl}_{3}\right) \delta=0.96\left(\mathrm{~A}, 3 \mathrm{H}, \mathrm{t},{ }^{3} J=6.7 \mathrm{~Hz}, \mathrm{CH}_{3}\right), 0.98\left(\mathrm{~B}, 3 \mathrm{H}, \mathrm{t},{ }^{3} J=6.7 \mathrm{~Hz}\right.$, 
$\left.\mathrm{CH}_{3}\right), 1.73-1.78(\mathrm{~A} / \mathrm{B}, \mathrm{m}, 2 \mathrm{H}), 3.76\left(3 \mathrm{H}, \mathrm{s}, \mathrm{OCH}_{3}, \mathrm{~A}\right), 3.79\left(3 \mathrm{H}, \mathrm{s}, \mathrm{OCH}_{3}, \mathrm{~B}\right)$, $4.14\left(\mathrm{OCH}, \mathrm{A}, 1 \mathrm{H}, \mathrm{t},{ }^{3} \mathrm{~J}=6.8 \mathrm{~Hz}\right), 4.40\left(\mathrm{OCH}, \mathrm{B}, 1 \mathrm{H}, \mathrm{t},{ }^{3} \mathrm{~J}=6.8 \mathrm{~Hz}\right), 6.79(\mathrm{~A}, 2 \mathrm{H}$, d, $\left.{ }^{3} J=8.4 \mathrm{~Hz}\right), 6.86\left(\mathrm{~B}, 2 \mathrm{H}, \mathrm{d},{ }^{3} J=8.4 \mathrm{~Hz}\right), 7.15\left(\mathrm{~A} / \mathrm{B}, 2 \mathrm{H}, \mathrm{d},{ }^{3} J=8.4 \mathrm{~Hz}\right) ;{ }^{13} \mathrm{C}$ $\operatorname{NMR}\left(67.8 \mathrm{MHz}, \mathrm{CDCl}_{3}\right): \delta=9.8[3]\left(\mathrm{B},+, \mathrm{CH}_{3}\right), 10.4$ [7] $\left(\mathrm{A},+, \mathrm{CH}_{3}\right), 29.6$ [0] $(\mathrm{A},-), 31.2[6](\mathrm{B},-), 55.2[1]\left(+, \mathrm{OCH}_{3}\right), 79.1[9](\mathrm{B},+, \mathrm{CH}), 80.0[2](\mathrm{A},+$, $\mathrm{CH}), 113.3$ [2] (A, 2C, +, CH), 113.5 [7] (B, 2C, +, CH), 127.9 [2] (B, 2C, +, CH), 128.2 [7] ( $\mathrm{A}, 2 \mathrm{C},+, \mathrm{CH}), 134.9$ [5] $\left(\mathrm{A}, \mathrm{C}_{\text {quat }}\right), 135.2$ [2] (B, $\left.\mathrm{C}_{\text {quat }}\right), 158.5$ [5] (B, $\left.\mathrm{C}_{\text {quat }}\right), 158.8$ [7] (A, $\left.\mathrm{C}_{\text {quat }}\right)$ ppm; MS (EI, $\left.70 \mathrm{eV}\right): \mathrm{m} / z(\%)=314\left(\mathrm{M}^{+}, 3.5\right), 285,149$ (100). HRMS Found: 314.1880. Calcd. for $\mathrm{C}_{20} \mathrm{H}_{26} \mathrm{O}_{3}: 314.1882$.

Bis(cinnamyl) ether(21b) [29] [30]-slowly crystallizing oil (Lit. mp. $43^{\circ} \mathrm{C}$ [26]); IR (neat) $v 3020,1600,1495,1450,1215,760 ;{ }^{1} \mathrm{H}$ NMR $\left(270 \mathrm{MHz}, \mathrm{CDCl}_{3}\right)$ $\delta=4.20\left(4 \mathrm{H}, \mathrm{dd},{ }^{3} J=6.3 \mathrm{~Hz}, J=1.5 \mathrm{~Hz}\right), 6.32\left(2 \mathrm{H}, \mathrm{td},{ }^{3} J=16.0 \mathrm{~Hz},{ }^{3} J=6.3 \mathrm{~Hz}\right)$, $6.65\left(2 \mathrm{H}, \mathrm{d},{ }^{3} \mathrm{~J}=16.0 \mathrm{~Hz}\right), 7.22-7.43(10 \mathrm{~m}) ;{ }^{13} \mathrm{C} \mathrm{NMR}\left(67.8 \mathrm{MHz}, \mathrm{CDCl}_{3}\right) \delta=$ 70.8 (2C, OCH2), 126.1 (2C, $2 \mathrm{CH}), 126.6(4 \mathrm{C}, 4 \mathrm{CH}), 127.9(2 \mathrm{C}, 2 \mathrm{CH}), 128.7$ (4C, 4CH), $132.7(2 \mathrm{C}, 2 \mathrm{CH}), 136.9\left(2 \mathrm{C}, 2 \mathrm{C}_{\text {quat }}\right) ; \mathrm{MS}(70 \mathrm{eV}) \mathrm{m} / z(\%) 250\left(\mathrm{M}^{+}\right)$ (13).

Preparation of styrene derivatives by eluting the corresponding arylalkyl ethers on silica gel with hexane-Methoxystyrene (24) [31]. Bis-1-(4-methoxyphenyl)ethyl ether (19a, $1.27 \mathrm{~g}, 4.4 \mathrm{mmol})$ was placed on silica gel (40 g) in a glass column and eluted continuously with hexane. After evaporation of the eluate, 4-methoxystyrene $(24,650 \mathrm{mg}, 55 \%)$ was isolated as a colorless oil; IR $\left(\right.$ neat $\left./ \mathrm{cm}^{-1}\right) v=3084,3004,2956,2834,1609,1510,1459,1319,1300,1250,1175$, 1113, 1038, 990, 901, $836 \mathrm{~cm}^{-1} ;{ }^{1} \mathrm{H}$ NMR $\left(270 \mathrm{MHz}, \mathrm{CDCl}_{3}\right): \delta=3.81(3 \mathrm{H}, \mathrm{s}$, $\left.\mathrm{OCH}_{3}\right), 5.12\left(1 \mathrm{H}, \mathrm{dd},{ }^{3} J=11.1 \mathrm{~Hz},{ }^{3} J=2.8 \mathrm{~Hz}\right), 5.60\left(1 \mathrm{H}, \mathrm{dd},{ }^{3} J=17.3 \mathrm{~Hz},{ }^{3} J=\right.$ $2.8 \mathrm{~Hz}), 6.66\left(1 \mathrm{H}, \mathrm{dd},{ }^{3} J=17.3 \mathrm{~Hz},{ }^{3} J=11.1 \mathrm{~Hz}\right), 6.86\left(2 \mathrm{H}, \mathrm{d},{ }^{3} J=8.9 \mathrm{~Hz}\right), 7.33$ $\left(2 \mathrm{H}, \mathrm{d},{ }^{3} J=8.9 \mathrm{~Hz}\right) \mathrm{ppm} ; \delta_{\mathrm{C}}\left(67.8 \mathrm{MHz}, \mathrm{CDCl}_{3}\right) 55.3\left(+, \mathrm{OCH}_{3}\right), 111.6(+, \mathrm{CH})$, $113.9(2 \mathrm{C},+, \mathrm{CH}), 127.4(2 \mathrm{C},+, \mathrm{CH}), 130.5\left(\mathrm{C}_{\text {quat }}\right), 136.2(+, \mathrm{CH}), 159.4\left(\mathrm{C}_{\text {quat }}\right)$ ppm; MS (70 eV) $\mathrm{m} / z(\%) 134\left(\mathrm{M}^{+}, 100\right), 119$ (38), 91 (45). The eluant hexane was reused after distillation.

Also, the following alkoxyphenylalkenes were prepared from crude bis(1-(4-alkoxyphenyl)propyl ethers:

(E) -1 -(4-Dodecyloxyphenyl) propene (25a): slowly crystallizing oil; ${ }^{1} \mathrm{H}$ NMR $\left(270 \mathrm{MHz}, \mathrm{CDCl}_{3}\right): \delta=0.88\left(3 \mathrm{H}, \mathrm{t},{ }^{3} J=7.0 \mathrm{~Hz}\right), 0.97\left(3 \mathrm{H}, \mathrm{t},{ }^{3} J=6.8 \mathrm{~Hz}\right), 1.27-1.49$ $(18 \mathrm{H}, \mathrm{m}), 1.78(2 \mathrm{H}, \mathrm{m}), 3.94\left(2 \mathrm{H}, \mathrm{t},{ }^{3} \mathrm{~J}=6.8 \mathrm{~Hz}\right), 6.04\left(1 \mathrm{H}, \mathrm{dq},{ }^{3} J=15.6 \mathrm{~Hz},{ }^{3} J=\right.$ $6.6 \mathrm{~Hz}), 6.30\left(1 \mathrm{H}, \mathrm{dd},{ }^{3} J=15.6 \mathrm{~Hz},{ }^{4} J=2.0 \mathrm{~Hz}\right), 6.82\left(2 \mathrm{H}, \mathrm{d},{ }^{3} J=8.9 \mathrm{~Hz}\right), 7.28$ $\left(2 \mathrm{H}, \mathrm{d},{ }^{3} \mathrm{~J}=8.9 \mathrm{~Hz}\right)$ ppm; MS (EI, $\left.70 \mathrm{eV}\right) \mathrm{m} / z(\%)=303\left(\mathrm{MH}^{+}, 5\right), 302\left(\mathrm{M}^{+}, 10\right)$. HRMS Found: 302.2607. Calcd. for $\mathrm{C}_{21} \mathrm{H}_{34} \mathrm{O}: 302.2610$.

(E)-1-(4-Ethoxyphenyl)propene (25b) [32]: M.p. $62{ }^{\circ} \mathrm{C} ;{ }^{1} \mathrm{H}$ NMR $(270 \mathrm{MHz}$, $\left.\mathrm{CDCl}_{3}\right): \delta=1.35\left(3 \mathrm{H}, \mathrm{t},{ }^{3} J=6.8 \mathrm{~Hz}\right), 1.83\left(3 \mathrm{H}, \mathrm{d},{ }^{3} J=6.6 \mathrm{~Hz},{ }^{4} J=2.0 \mathrm{~Hz}\right), 3.92$ $\left(2 \mathrm{H}, \mathrm{q},{ }^{3} J=6.8 \mathrm{~Hz}\right), 6.05\left(1 \mathrm{H}, \mathrm{dq},{ }^{3} J=15.6 \mathrm{~Hz},{ }^{3} J=6.6 \mathrm{~Hz}\right), 6.32\left(1 \mathrm{H}, \mathrm{dd},{ }^{3} J=15.6\right.$ $\left.\mathrm{Hz},{ }^{4} J=2.0 \mathrm{~Hz}\right), 6.84\left(2 \mathrm{H}, \mathrm{d},{ }^{3} J=8.9 \mathrm{~Hz}\right), 7.30\left(2 \mathrm{H}, \mathrm{d},{ }^{3} J=8.9 \mathrm{~Hz}\right) \mathrm{ppm}$; MS (EI, $70 \mathrm{eV}): m / z(\%)=162\left(\mathrm{M}^{+}, 100\right), 133$ (84). HRMS Found: 162.1043. Calcd. for $\mathrm{C}_{11} \mathrm{H}_{14} \mathrm{O}: 162.1045$. 
(E)-1-(4-Butoxyphenyl) propene (25c): oil; ${ }^{1} \mathrm{H} \mathrm{NMR}\left(270 \mathrm{MHz}, \mathrm{CDCl}_{3}\right): \delta=$ $0.97\left(3 \mathrm{H}, \mathrm{t},{ }^{3} J=6.8 \mathrm{~Hz}\right), 1.36\left(3 \mathrm{H}, \mathrm{t},{ }^{3} J=6.8 \mathrm{~Hz}\right), 1.40(2 \mathrm{H}, \mathrm{m}), 1.73(2 \mathrm{H}, \mathrm{m}), 3.94$ $\left(2 \mathrm{H}, \mathrm{t},{ }^{3} J=6.8 \mathrm{~Hz}\right), 6.04\left(1 \mathrm{H}, \mathrm{dq},{ }^{3} J=15.6 \mathrm{~Hz},{ }^{3} J=6.6 \mathrm{~Hz}\right), 6.30\left(1 \mathrm{H}, \mathrm{dd},{ }^{3} J=\right.$ $\left.15.6 \mathrm{~Hz},{ }^{4} J=2.0 \mathrm{~Hz}\right), 6.82\left(2 \mathrm{H}, \mathrm{d},{ }^{3} J=8.9 \mathrm{~Hz}\right), 7.28\left(2 \mathrm{H}, \mathrm{d},{ }^{3} J=8.9 \mathrm{~Hz}\right)$ ppm; MS (EI, $70 \mathrm{eV}): m / z(\%)=190\left(\mathrm{M}^{+}, 49\right), 134(100), 133$ (48). HRMS Found: 190.1361. Calcd. for $\mathrm{C}_{13} \mathrm{H}_{18} \mathrm{O}: 190.1358$.

(E)-1-(4-Pentoxyphenyl)propene(25d): oil; ${ }^{1} \mathrm{H} \mathrm{NMR}\left(270 \mathrm{MHz}, \mathrm{CDCl}_{3}\right): \delta=$ $0.96\left(3 \mathrm{H}, \mathrm{t},{ }^{3} \mathrm{~J}=6.8 \mathrm{~Hz}\right), 1.27-1.40(7 \mathrm{H}, \mathrm{m}), 1.70(2 \mathrm{H}, \mathrm{m}), 3.93\left(2 \mathrm{H}, \mathrm{t},{ }^{3} \mathrm{~J}=6.8\right.$ $\mathrm{Hz}), 6.04\left(1 \mathrm{H}, \mathrm{dq},{ }^{3} J=15.4 \mathrm{~Hz},{ }^{3} J=6.6 \mathrm{~Hz}\right), 6.30\left(1 \mathrm{H}, \mathrm{dd},{ }^{3} J=15.4 \mathrm{~Hz},{ }^{4} J=1.8\right.$ $\mathrm{Hz}), 6.82\left(2 \mathrm{H}, \mathrm{d},{ }^{3} \mathrm{~J}=8.9 \mathrm{~Hz}\right), 7.29\left(2 \mathrm{H}, \mathrm{d},{ }^{3} J=8.9 \mathrm{~Hz}\right) \mathrm{ppm}$; MS (EI, $\left.70 \mathrm{eV}\right): \mathrm{m} / \mathrm{z}$ $(\%)=204\left(\mathrm{M}^{+}, 46\right), 134(100)$. HRMS Found: 204.1511. Calcd. for $\mathrm{C}_{14} \mathrm{H}_{20} \mathrm{O}$ : 204.1514.

(E) -1 -(4-Hexyloxyphenyl)propene(25e): oil; IR (neat $\left./ \mathrm{cm}^{-1}\right) \quad v=3060,3022$, 2921, 2856, 1607, 1511, 1472, 1304, 1281, 1245, 1175, 1019, 962, 937, 838, 786 $\mathrm{cm}^{-1} ;{ }^{1} \mathrm{H} \mathrm{NMR}\left(270 \mathrm{MHz}, \mathrm{CDCl}_{3}\right): \delta=0.97\left(3 \mathrm{H}, \mathrm{t},{ }^{3} \mathrm{~J}=6.8 \mathrm{~Hz}\right), 1.26-1.40(9 \mathrm{H}$, m), $1.70(2 \mathrm{H}, \mathrm{m}), 3.94\left(2 \mathrm{H}, \mathrm{t},{ }^{3} J=6.8 \mathrm{~Hz}\right), 6.06\left(1 \mathrm{H}, \mathrm{dq},{ }^{3} J=15.4 \mathrm{~Hz},{ }^{3} J=6.6\right.$ $\mathrm{Hz}), 6.30\left(1 \mathrm{H}, \mathrm{dd},{ }^{3} J=15.4 \mathrm{~Hz},{ }^{4} J=1.8 \mathrm{~Hz}\right), 6.82\left(2 \mathrm{H}, \mathrm{d},{ }^{3} J=8.9 \mathrm{~Hz}\right), 7.30(2 \mathrm{H}$, $\left.\mathrm{d},{ }^{3} \mathrm{~J}=8.9 \mathrm{~Hz}\right) ;{ }^{13} \mathrm{C} \mathrm{NMR}\left(67.8 \mathrm{MHz}, \mathrm{CDCl}_{3}\right): \delta=14.0,18.4,22.6,25.7,29.3,31.6$, 68.0, 114.5, 123.3, 126.8, 130.4, 130.6, $158.2 \mathrm{ppm}$; MS (EI, $70 \mathrm{eV}): \mathrm{m} / \mathrm{z}(\%)=218$ $\left(\mathrm{M}^{+}, 38\right), 134$ (100). HRMS Found: 218.1667. Calcd. for $\mathrm{C}_{15} \mathrm{H}_{22} \mathrm{O}: 218.1671$.

\section{Results and Discussion}

When 4-alkoxybenzyl alcohols 3 were heated in the presence of a catalytic amount of $\mathrm{Pt}$ on carbon at $135^{\circ} \mathrm{C}$, bis(4-alkoxybenzyl) ethers 16 formed (Scheme 5). The author has monitored this reaction and has found that in the case of 4-methoxybenzyl alcohol (3e) with $0.12 \mathrm{~mol} \%$ Pt over $50 \%$ of the substrate was consumed after $90 \mathrm{~min}$ and that the reaction was effectively complete after $6 \mathrm{~h}$, with less than $5 \%$ substrate remaining. 4-Methoxybenzaldehyde (2f) as the major by-product was produced in the very early stages of the reaction, and there was some small turn-over to 4-methoxybenzoic acid as the reaction proceeded. It must be noted that the reaction was carried out under normal atmosphere. Bis(4-methoxybenzyl) ether (16a) could be isolated in 95\% yield from the reaction mixture. Even lower catalyst loadings, as low as $0.04 \mathrm{~mol} \%$, can be used for the reaction, although this necessitates a considerable longer reaction time. The use of $10 \mathrm{w} \%$-Pt-5 w\%-Fe nanoparticles immobilized on carbon nanofibers gave bis(4-methoxybenzyl) ether (16a) in 77\% yield after $6 \mathrm{~h}$, albeit with $4 \%$ methoxybenzaldehyde (2f), with the remainder being unreacted material. With 20 w\% Pt-Ru nanoparticles immobilized on carbon nanofibers, 4-methoxybenzaldehyde (2f) can be isolated in $20 \%$ yield after $6 \mathrm{~h}$ with $64 \%$ of the dibenzyl ether (16a) as product Figure 1). The oxidizing power of the latter catalyst can be seen in the oxidation of benzhydrols $17 \mathrm{a}$ and $17 \mathrm{~b}$ in air saturated diphenyl ether to 18ain $55 \%$ yield [ $1 \mathrm{~h}](88 \%[3 \mathrm{~h}])$ and to $18 \mathrm{~b}$ in $52 \%$ yield [1h], respectively (Scheme 6). 
<smiles>[R9]c1ccc(CO)cc1</smiles>

3<smiles>COc1ccc(CO)cc1OC</smiles>

$3 e$<smiles>COc1ccc(COCc2ccc(OC)c(OC)c2)cc1OC</smiles><smiles>Cc1ccc(CO)cc1</smiles><smiles>Cc1ccc(COCc2ccc(C)cc2)cc1</smiles>

$16 f(35 \%)$

Scheme 5. Pt/C catalyzed solventless transformation of benzyl alcohols 3 to dibenzyl ethers 16.<smiles>OC(c1ccccc1)c1ccccc1</smiles>

$17 a$<smiles>Cc1ccc(C(O)c2ccc(C)cc2)cc1</smiles>

17b

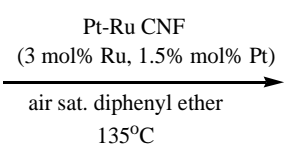

$135^{\circ} \mathrm{C}$<smiles>O=C(c1ccccc1)c1ccccc1</smiles>

$18 a$

(1h, 55\%; 3h, 88\%)<smiles>Cc1ccc(C(=O)c2ccc(C)cc2)cc1</smiles>

18b

(1h, 52\%)

Scheme 6. Solventless oxidation of benzhydrols 17 to benzophenones 18 with Pt-Ru CNF as catalyst.

Similar results of etherification over $\mathrm{Pt} / \mathrm{C}$ could be found with other 4-alkoxybenzyl alcohols 3, which formed the corresponding bis(4-alkoxybenzyl) ethers 16 under the above conditions. When 4-pentoxybenzyl alcohol (3d) was heated in a neat state over activated carbon (granular, 4-14 mesh, Aldrich) at $135^{\circ} \mathrm{C}$ for $3 \mathrm{~h}$, i.e., in the absence of $\mathrm{Pt} / \mathrm{C}$, no reaction could be detected. As mentioned above, alkoxybenzyl alcohols do form ethers in the absence of metal catalysts, such as when heated in DMSO at $175^{\circ} \mathrm{C}$ [9]. DMSO, however, must be viewed not only as a solvent in the reaction but also as a participating species, i.e., as a 
reagent, by activating the alcohol [9]. A similar participation of DMSO has been forwarded in the thermal dehydration of alcohols [33] [34].

Reactions of the benzyl alcohols 3 in the presence of $\mathrm{Pd} / \mathrm{C}$ also led to dibenzyl ethers 16 as the main products. Here, however, the oxidation of benzyl alcohols 3 to benzaldehydes 2 is much more pronounced than with $\mathrm{Pt} / \mathrm{C}$. The oxidation of alcohols over $\mathrm{Pd} / \mathrm{C}$ is well known and liquid oxidation of benzyl alcohol itself in the presence of Pd doped carbon materials has been communicated previously [35] [36]. Also, the outcome of the reaction-etherification vs. oxidation was found to be very dependent on the substitution pattern of the benzyl alcohol used as substrate. Thus, while 4-alkoxybenzyl alcohols 3a-e predominately gave the bis(4-alkoxybenzyl) ethers 16 in good yield, the less electron rich 4-alkylbenzyl alcohols such as $\mathbf{3 f}$ gave the corresponding ethers in much lower yields. The reactions in the presence of commercial $\mathrm{Pd}$ on carbon $\left(135^{\circ} \mathrm{C}\right.$, solvent free, $0.26 \mathrm{~mol} \% \mathrm{Pd}$ ) provided a mixture of compounds with benzaldehydes and products derived from benzaldehydes very much in evidence. Also, over Pt/C, 4-alkylbenzyl alcohols reacted very sluggishly under otherwise identical conditions (Scheme 5).

Next, we turned our attention to the reactivity of 4-alkoxyphenylalkyl alcohols 7over Pt/C and Pd/C. The preparation of bis(phenylalkyl) ethers have been reported from the corresponding alcohols by acid catalyzed etherification [1]. Where 4-toluenesulfonic acid has been used as catalyst, the reaction times are long $(8 \mathrm{~h})$ and the respective dehydration product, styrene, is formed in noticeable amounts (10\%) [1]. Potassium bisulfate has also been used as catalyst in the reaction with high-boiling aromatic solvents such as toluene and 1,2,4-trimethylbenzene, with bis(phenylalkyl) ethers forming in moderate yield (51\%) [1]. One example of a liquid phase etherification with potassium bisulfate has also been reported [1]. A bis(phenylalkyl) ether is known to form in small amounts in the Swern oxidation of 1-(4-anisyl)ethanol [20]. Also, bis(phenylalkyl) ethers are formed from the corresponding alcohols in DMSO at $175^{\circ} \mathrm{C}$ (see above) [9].

When 4-alkoxyphenylalkyl alcohols 7 and 9 were heated at $135^{\circ} \mathrm{C}$ in the presence of a catalytic amount of Pt/C (at 0.12 - $0.15 \mathrm{~mol} \% \mathrm{Pt}$ ), bis(4-alkoxypheny-lalkyl) ethers 19 were obtained. Initially, the bis(phenylalkyl) ethers 19 were formed in almost equal amounts of $D L$ - and meso-form (Scheme 7). When the reaction was continued after the consumption of the alkoxyphenylalkyl alcohol, the ratio $D L$-isomer/meso-isomer changed gradually towards the $D L$-isomer as determined by ${ }^{1} \mathrm{H}$ NMR spectroscopic analysis during the course of reaction. It must also be noted that the catalysts used facilitate the production of styrenes, most likely mainly via the meso-bis(phenylalkyl) ethers, and prolonged reaction can lead to more styrene. The reaction with $\mathrm{Pt} / \mathrm{C}$ led to small amounts of styrenes $24 / 25$ and to traces of the arylketones as oxidation products, while $\mathrm{Pd} / \mathrm{C}$ (at 0.15 $\mathrm{mol} \%$ and $0.26 \mathrm{~mol} \% \mathrm{Pd}$ ) led to comparably larger amounts of arylketones, but only to traces of styrenes $24 / 25$ as side products, when the reactions were stopped after the consumption of the benzylalkyl alcohol. Often, a quicker 
<smiles>COc1ccc(C(C)O)cc1</smiles>

9a<smiles>Cc1ccc(C(C)O)cc1</smiles>

9b<smiles>CCC(O)c1ccc(OC)cc1</smiles>

$7 \mathbf{b}$

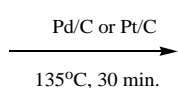<smiles>COc1ccc(C(C)OC(C)c2ccc(OC)cc2)cc1</smiles>

19a<smiles>Cc1ccc(C(C)OC(C)c2ccc(C)cc2)cc1</smiles>

19b<smiles>CCC(OC(CC)c1ccc(OC)cc1)c1ccc(OC)cc1</smiles>

19c

(78\% [Pd]; 83\% [Pt])

Scheme 7. Pd/C and Pt/C catalyzed ether formation with phenylalkyl alcohols (7/9).

turnover of starting material to product could be seen over Pd/C than over Pt/C at comparable catalyst loading.

As for benzyl alcohols, the reaction over $\mathrm{Pt} / \mathrm{C}$ and $\mathrm{Pd} / \mathrm{C}$ is substrate dependent. Thus, it was found that alkylated phenylethyl alcohols gave more acetophenone as side product with a variety of $\mathrm{Pd} / \mathrm{C}$ and $\mathrm{Pt} / \mathrm{C}$ (incl. those used above) and $\mathrm{Pt}-\mathrm{Ru} / \mathrm{C}$ catalysts under conditions identical to those described above for the preparation of the ethers. Also, the steric characteristics of the aryl group of the substrates exert an influence on the reaction. The reaction of 3,5-dimethyl derivatives (not shown here) proceeded much more slowly than that of the demethyl analogs. It must be noted that the reaction temperature could be lowered to $100^{\circ} \mathrm{C}$ in many cases and even as low as to $80^{\circ} \mathrm{C}$ for the transformation of the alcohols to the corresponding ethers in $4 \mathrm{~h}$ to $11 \mathrm{~h}$.

When different Pd catalysts on solid supports were screened, it was seen that also the use of commercial Lindlar catalyst ( $\mathrm{Pd}$ on $\mathrm{CaCO}_{3}$ support, poisoned with lead, $0.2 \mathrm{~mol} \% \mathrm{Pd}$ ) leads to the etherification of substrates at $135^{\circ} \mathrm{C}$ (Scheme 8). Here, the reaction is influenced greatly by the size and electronic character of the substrate. Thus, the smaller 4-methoxyphenyl ethyl alcohol 9a and non-substituted $D L$-phenylethyl alcohol underwent rapid oxidation to the corresponding acetophenones under the conditions, while 4-methylphenyl ethyl alcohol (9b) reacted sluggishly, giving predominately oxidized product. On the other hand, 4-methoxybenzyl alcohol (3d) underwent etherification in the presence of Lindlar catalyst (at $0.2 \mathrm{~mol} \%$ ) to give $16 \mathrm{a}$ in $85 \%$ yield after $11 \mathrm{~h}$ (Scheme 8$)$. Under similar conditions $\left(135^{\circ} \mathrm{C}\right)$, but shorter reaction time $(3 \mathrm{~h})$, the use of Pearlman catalyst equally led to ether 16a (49\%) after $3 \mathrm{~h}$, but also to a number of side products, such as to 4-methoxybenzaldehyde (2f) (8\%) and to 4-methox-ybenzyl 4-methoxybenzoate (20) (5\%) (Scheme 8). 

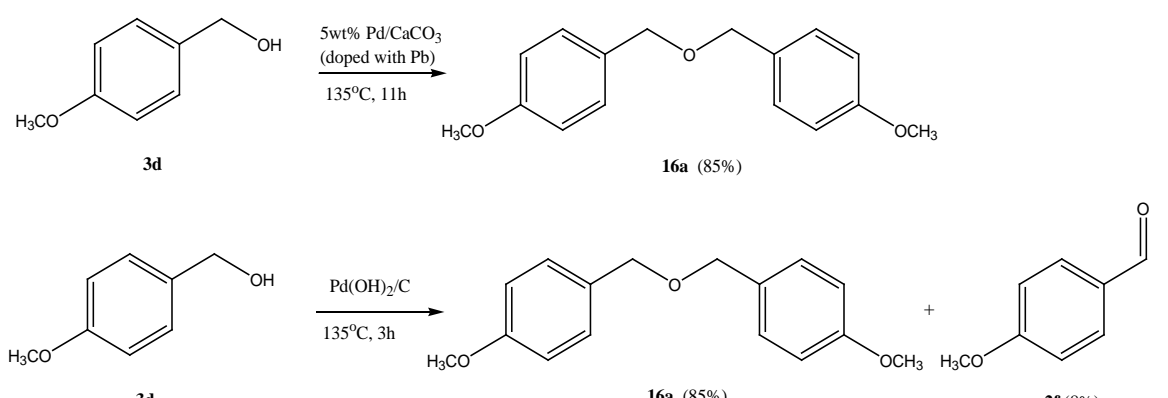

$3 d$

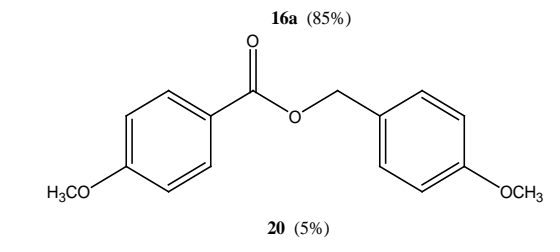

$2 \mathbf{f}(8 \%)$

Scheme 8. Solventless etherfication of benzyl alcohols over Lindlar and Pearlman catalysts.

Lastly, cinnamyl alcohols 11 were reacted at $135^{\circ} \mathrm{C}$ over $\mathrm{Pd} / \mathrm{C}$ (at $0.26 \mathrm{~mol} \%$ Pd). 4-Methoxycinnamyl alcohol(11a) gave the corresponding bis(cinnamyl) ether 21a in fair yield, however, non-substituted cinnamyl alcohol $11 \mathrm{~b}$ gave ether $21 \mathrm{~b}$ in poor yield (Scheme 9). It is known that cinnamyl alcohol and phenylalkenols (see below) can oxidize readily over Pd/C. In the cases, where oxidation takes place, the abstracted hydrogen leads to Pd catalyzed hydrogenation of the olefinic bond of the cinnamaldehydes and phenylbutenones formed as oxidation side products, giving phenylbutanones and phenylpropanals as further side products. Similar hydrogenation reactions concurrent with the dehydrogenative oxidation of fluorinated cinnamyl alcohols over Pd/C have been found as main pathways leading to fluorinated phenylbutanones as side products. Again, the outcome of the reactions was dependent on the substitution pattern of the phenyl group in the substrates. Thus, 3,4,5-trifluorophenylbutenol 15a underwent the etherification in good yield, where the meso- and DL-isomers could be separated by column chromatography. On the other hand, 2,4-difluorophenylbutenol $15 \mathrm{~b}$ gave the corresponding ether in poor yield with a number of sideproducts 23a-23e, which could be separated and identified (Scheme 10).

While the mechanism of this general type of etherification reaction is not clear, in the $\operatorname{Pd}(0)$ mediated transformations, a reactive $\mathrm{Pd}(\mathrm{II})$ species [37] may in fact be involved. The formal oxidation of the $\operatorname{Pd}(0)$ species may be brought about by the benzyl alcohol/cinnamyl alcohol itself. Although styrenes form as side products, more olefinic and oligomeric products would be expected at the elevated temperature used, in case of a pure $\mathrm{S}_{\mathrm{N}} 1$ mechanism, which has been determined to occur in the equivalent etherification of benzyl alcohols with $\mathrm{Pd}(\mathrm{II})$ catalysts in nitromethane at much lower temperatures $\left(50^{\circ} \mathrm{C}\right)[10]$. Thus, it may well be that a mixture of $\mathrm{S}_{N}$ mechanisms occur with the substrate bound to the metal. A tentative schematic mechanism is shown in Scheme 11.

Bis(phenylalkyl) ethers 19 are sensitive to long exposure to silica gel, where especially the meso-isomer forms the corresponding styrene 25 (Scheme 12). 
<smiles>[R]c1ccc(/C=C/CO)cc1</smiles>

$\mathrm{R}=\mathrm{OCH}_{3}(\mathbf{1 1} \mathbf{a}), \mathrm{H}(\mathbf{1 1} \mathbf{b})$

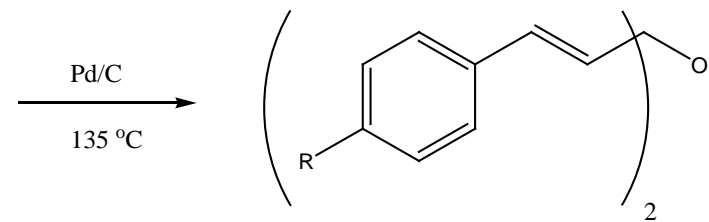

$\mathrm{R}=\mathrm{OCH}_{3}(\mathbf{2 1 a})$ [75\%], $\mathrm{H}(\mathbf{2 1 b})[32 \%]$

Scheme 9. Preparation of bis(cinnamyl) ethers 21.
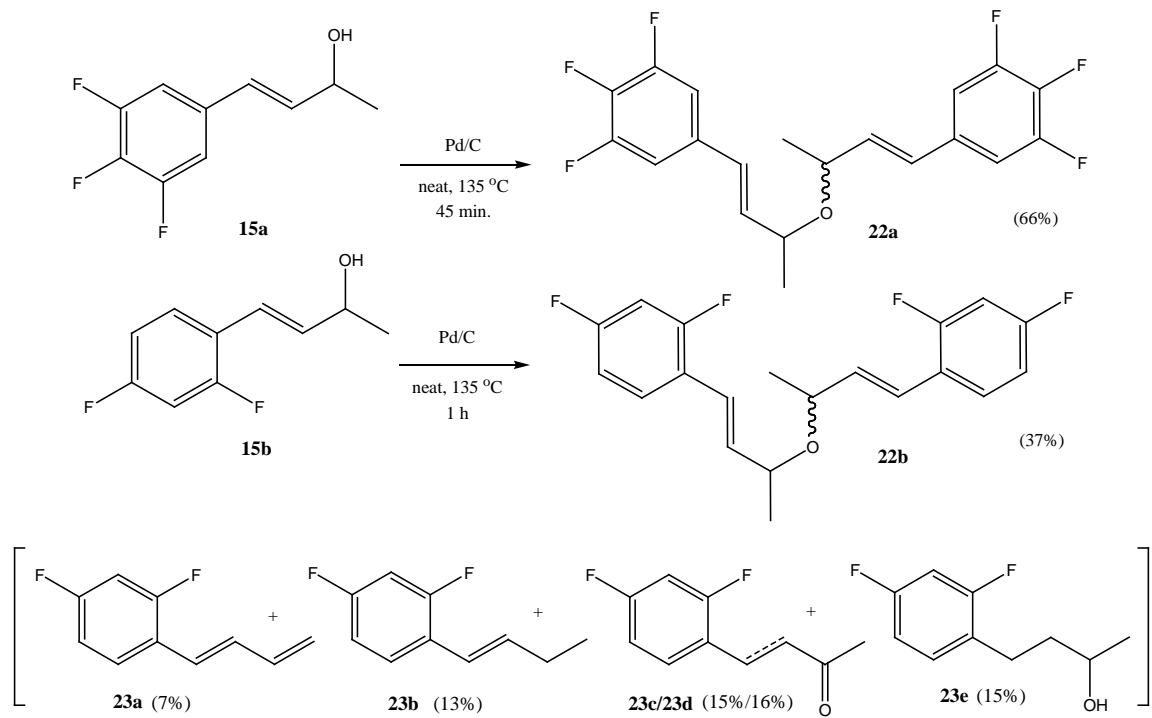

Scheme 10. Ether formation using fluorinated 4-phenylbut-3-en-2-ols 15 as substrate.
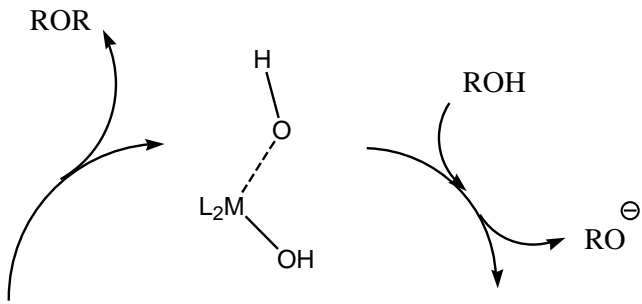

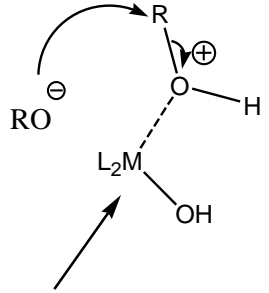

$\mathrm{M}=\mathrm{Pd}$ or $\mathrm{Pt}$

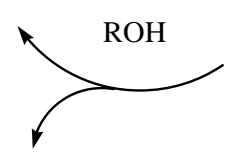

$\mathrm{H}_{2} \mathrm{O}$

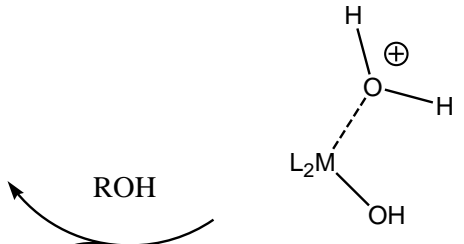

Scheme 11. Tentative reaction mechanism.

Thus, appreciable amounts of styrene are obtained by submitting the bis(phenylalkyl) ethers 19 to a further column chromatography on silica gel with hexane as eluant. Previously, an acid catalyzed process of preparing styrenes 25 from bis(phenylalkyl) ethers 19 , albeit at between $150^{\circ} \mathrm{C}-220^{\circ} \mathrm{C}$ under reduced pressure, had been patented [1]. As acids, methanesulfonic acid, ammonium 


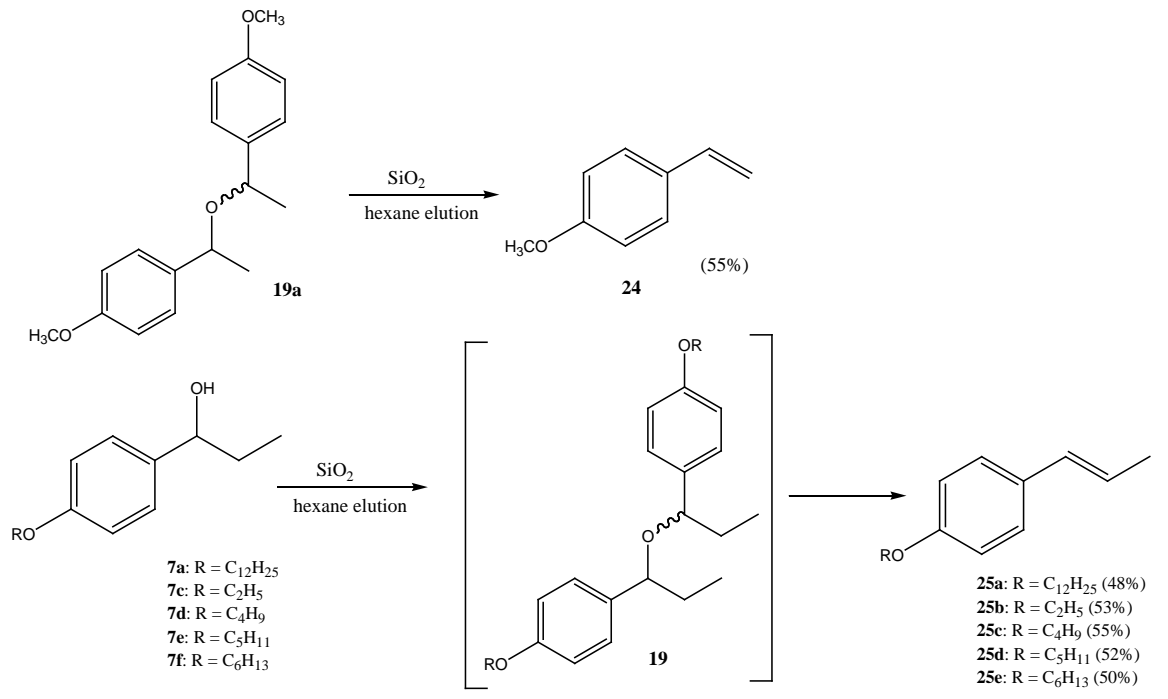

Scheme 12. Transformation of ethers 19 to styrene derivatives 25 on silica gel.

bisulfate and phosphoric acid had been used [1]. If needed to be purified, bis(phenylalkyl) ethers are stable enough to be eluted over Florisil (Wako). They can also be purified over silica gel by flash column chromatography (hexane/ ether $10: 1$ to hexane/ether/ $\mathrm{CHCl}_{3}$ 5:1:1). In the present study, usually the $D L$ and meso-isomers 19 were not separated from each other. When, attempting to obtain styrene derivatives it was possible to subject the reaction mixture of the etherification reactions, composed mainly of 19 , directly to an elution from silica gel with hexane.

\section{Conclusion}

In the present manuscript, it could be shown that alkoxybenzyl alcohols undergo etherification when heated over Pt/C. The use of $\mathrm{Pd} / \mathrm{C}$ and $\mathrm{Pt}-\mathrm{Ru}$ on carbon nanofiber (CNF) is also possible for this transformation, however, side-products stemming from alcohol oxidation are observed in this case. Interestingly, also Lindlar catalyst ( $\mathrm{Pd}$ on $\mathrm{CaCO}_{3}$, poisoned with $\mathrm{Pb}$ ) gave acceptable results for this reaction. Reactions of 1-phenylalkan-1-ols lead to etherification, when the neat substrates are heated over $\mathrm{Pd} / \mathrm{C}$. The resulting ethers, which are mixtures of $D L$ - and meso-isomers are acid labile and can be transformed to styrene derivatives by immobilizing them on silica gel in a glass column and eluting the olefinic product with hexane.

\section{Acknowledgements}

Some of this work has been carried out within the framework of a CREST program (2005-2007). Financial support from the Japan Science and Technology Corporation (JST) is gratefully acknowledged. The author thanks Prof. Dr. I. Mochida and Prof. Dr. S. H. Yoon for generation donations of $10 \mathrm{w} \%-\mathrm{Pt}-5$ w\%-Fe@CNF and Pt-Ru@CNF. The mass spectrometric measurements have been carried out by Ms. Y. Tanaka, Institute of Materials Chemistry and Engi- 
neering, Kyushu University. Elemental analyses were performed at the Center of Instrumental Analysis, Hakozaki Campus, Kyushu University.

\section{References}

[1] Aslam, M., Smith, B.L. and Kvakovszky, G. (1993) Process of Preparation of Substituted Styrenes. US Patent No. 5194672. Chemical Abstracts, 119, 139969.

[2] Greene, T.W. and Wuts, P.G.M. (1999). Protective Groups in Organic Synthesis. 3rd Edition, John Wiley \& Sons, Inc., New York, 76-76.

https://doi.org/10.1002/0471220574

[3] Kim, S., Chung, K.N. and Yang, S. (1987) Direct Synthesis of Ethers via Zinc Chloride-Mediated Etherification of Alcohols in Dichloroethane. Journal of Organic Chemistry, 52, 3917-3919. https://doi.org/10.1021/jo00226a036

[4] deMico, A., Margarita, R. and Piancatelli, G. (1995) Electrophilic Assistance for Cross-Coupling Reactions-A Simple Synthesis of Mixed Allylic Ethers. Tetrahedron Letters, 36, 2679-2680. https://doi.org/10.1016/0040-4039(95)00332-7

[5] Firouzabadi, H., Iranpoor, N. and Jafari, A.A. (2005) Facile Preparation of Symmetrical and Unsymmetrical Ethers from Their Corresponding Alcohols Catalyzed by Aluminumdodecatangstophosphate $\left(\mathrm{AlPW}_{12} \mathrm{O}_{40}\right)$, as a Versatile and a Highly Water Tolerant Lewis Acid. Journal of Molecular Catalysis A: Chemical, 227, 97-100. https://doi.org/10.1016/j.molcata.2004.09.078

[6] Ooi, T., Ichikawa, H., Itagaki, Y. and Maruoka, K. (2000) Efficient Catalytic Procedure for Etherification of Alcohols with MeAl(NTf2)(2). Heterocycles, 52, 575-578. https://doi.org/10.3987/COM-99-S99

[7] Salmón, M., Zavala, N., Cabrera, A., Cárdenas, J., Gaviño, R., Miranda, R. and Martínez, M. (1995) Aromatic-Substitution Reactions of Benzyl Derivatives with a Bentonite Clay. Journal of Molecular Catalysis A: Chemical, 104, L127-L129. https://doi.org/10.1016/1381-1169(95)00106-9

[8] Salmón, M., Zavala, N., Martínez, M., Miranda, R., Cruz, R., Cárdenas, J., Gaviño, R. and Cabrera, A. (1994) Cyclic And Linear Oligomerization Reaction of 3,4,5-Trimethoxybenzyl Alcohol with A Bentonite-Clay. Tetrahedron Letters, 35, 5797-5800. https://doi.org/10.1016/S0040-4039(00)78187-1

[9] Emert, J., Goldenberg, M., Chiu, G.L. and Valeri, A. (1977) Synthesis of Dibenzyl Ethers via the Dehydration of Benzylic Alcohols in Dimethyl Sulfoxide. Journal of Organic Chemistry, 42, 2012-2013. https://doi.org/10.1021/jo00431a042

[10] Miller, K.J. and Abu-Omar, M.M. (2003) Palladium-Catalyzed $S_{N} 1$ Reactions of Secondary Benzylic Alcohols: Etherification, Amination, and Thioetherification. European Journal of Organic Chemistry, 2003, 1294-1299. https://doi.org/10.1002/ejoc.200390185

[11] Bouquillon, S., Hénin, F. and Muzart, J. (2000) Critical Role of the Coordination Environment of Palladium Dichloride on the Course of Its Reaction with Secondary Benzylic Alcohols: Selective Oxidation or Etherification Catalysts. Organometallics, 19, 1434-1437. https://doi.org/10.1021/om990961q

[12] Derdar, K.F., Martin, J., Martin, C., Brégeault, J.-M. and Mercier, J. (1998) Cétonisation catalytique du méthyl-2-butène-3-ol-2 par des complexes du rhodium(III) ou du palladium(II). Journal of Organometallic Chemistry, 338, C21-C26. https://doi.org/10.1016/0022-328X(88)80515-1

[13] Muzart, J. (2005) Palladium-Catalysed Reactions of Alcohols. Part C: Formation of Ether Linkages. Tetrahedron, 61, 5955-6008. 
https://doi.org/10.1016/j.tet.2005.04.002

[14] Thiemann, T. (2007) Etherification of (E)-1,3-diaryl- and (E)-1,3-DiheteroarylProp-2-En-1-Ols with Primary and Secondary Alcohols over Platinum on Carbon. Journal of Chemical Research, 2007, 528-534. https://doi.org/10.3184/030823407X24775

[15] Thiemann, T. (2009) Etherification of Diarylmethanols and 1-Phenylalkan-1-ols Over Platinum on Carbon. Letters of Organic Chemistry, 6, 515-525. https://doi.org/10.2174/157017809789869564

[16] Kalviri, H.A., Petten, C.F. and Kerton, F.M. (2009) Catalytic Dehydrative Etherification and Chlorination of Benzyl Alcohols in Ionic Liquids. Chemical Communications, 45, 5171-5173. https://doi.org/10.1039/b909866f

[17] Tsuji, M., Kubokawa, M., Yano, R., Miyamae, N., Tsuji, T., Jun, M.S., Hong, S., Lim, S., Yoon, S.H. and Mochida, H. (2007) Fast Preparation of PtRu Catalysts Supported on Carbon Nanofibers by the Microwave-Polyol Method and Their Application to Fuel Cells. Langmuir, 23, 387-390. https://doi.org/10.1021/la062223u

[18] Juršić, B. (1988) Synthetic Application of Micellar Catalysis. Williamson’s Synthesis of Ethers. Tetrahedron, 44, 6677-6680. https://doi.org/10.1016/S0040-4020(01)90106-1

[19] Johnstone, R.A.W. and Rose, M.E. (1979) A Rapid, Simple, and Mild Procedure for Alkylation of Phenols, Alcohols, Amides and Acids. Tetrahedron, 35, 2169-2173. https://doi.org/10.1016/0040-4020(79)87035-0

[20] Thiemann, T. (2007) Solventless Wittig Olefination with Fluorinated Benzaldehydes. Journal of Chemical Research, 2007, 336-341. https://doi.org/10.3184/030823407X225464

[21] Thiemann, T., Tanaka, Y., Ideta, K. and Mataka, S. (2006) Solvent Reduced Wittig Olefination Reactions with Halo Containing Conjugated Phosphoranes. Central European Journal of Chemistry, 4, 403-427. https://doi.org/10.2478/s11532-006-0024-2

[22] Thiemann, T., Watanabe, M., Tanaka, Y. and Mataka, S. (2004) Solvent-Free Wittig Olefination with Stabilized Phosphoranes-Scope and Limitations. New Journal of Chemistry, 28, 578-584. https://doi.org/10.1039/B311894K

[23] Luche, J.-L., Rodriguez-Hahn, L. and Crabbé, P. (1978) Reduction of Natural Enones in the Presence of Cerium Trichloride. Journal of the Chemical Society, Chemical Communications, 14, 601-602. https://doi.org/10.1039/C39780000601

[24] Kalviri, H.A. and Kerton, F.M. (2011) Dehydration of Benzyl Alcohols in Phosphonium Ionic Liquids: Synthesis of Ethers and Alkenes. Advanced Synthesis \& Catalysis, 353, 3178-3186. https://doi.org/10.1002/adsc.201100445

[25] Lindgren, B.O. (1950) Veratryl Alcohol, Diveratryl Ether, and Veratryl-ethyl Ether as Lignin Models. Acta Chemica Scandinavica, 4, 1365-1374. https://doi.org/10.3891/acta.chem.scand.04-1365

[26] Barbero, M., Bazzi, S., Cadamuro, S. and Dughera, S. (2009) o-Benzenedisulfonimide as a Reusable Brønsted Acid Catalyst for Ritter-Type Reactions. European Journal of Organic Chemistry, 2009, 430-436. https://doi.org/10.1002/ejoc.200800931

[27] Yamato, T., Hu, J.Y. and Shinoda, N. (2007) Perfluorinated Sulfonic Acid Resin (Nafion-H) Catalysed Ritter Reaction of Benzyl Alcohols. Journal of Chemical Research, 2007, 641-643. https://doi.org/10.3184/030823407X262436

[28] Babu, G.A. and Ramasamy, P. (2008) Investigation of Crystal Growth, Structural, Optical, Dielectric, Mechanical and Thermal Properties of a Novel Organic Crystal: 
4, 4'-dimethylbenzophenone. Journal of Crystal Growth, 310, 3561-3567.

[29] Staab, H.A. and Wendel, K. (1960) Synthesen und Umsetzungen von Imidazoliden aromatischer Sulfonsäuren. Chemische Berichte, 93, 2902-2915. https://doi.org/10.1002/cber.19600931223

[30] Wagh, Y.S., Sawant, D.N., Tambada, P.J., Dhake, K.P. and Bhanage, B.M. (2011) $\mathrm{Pd}(\mathrm{OAc})_{2} / \mathrm{Dppf}$ as an Efficient and Highly Active Catalyst for the Allylation of Amines, Alcohols and Carboxylic Acids with 1-Phenyl-1-Propyne. Tetrahedron, 67, 2414-2421. https://doi.org/10.1016/j.tet.2011.02.008

[31] Ahmad, K., Thomas, N.F., Mukhtar, M.R., Noorbatcha, F., Weber, J.-F.F., Nafiah, M.A., Velu, S.S., Takeya, K., Morita, H., Lim C.G., Hadi, A. H.A. and Awang, K. (2009) A FeCl3-Promoted Highly Atropodiastereoselective Cascade Reaction: Synthetic Utility of Radical Cations in Indolostilbene Construction. Tetrahedron, 65, 1504-1516.

[32] Da Silva, J.C. and Marechal, E. (1974) Cationic Polymerization of Monomers of the Propenylbenzene Series. I. Preparation of the Monomers. Bulletin de la Societe Chimique de France, 5-6, 779-782.

[33] Traynelis, V.J., Hergenrother, W.I., Livingston, J.R. and Valicenti, J.A. (1962) Dehydration of Alcohols in Dimethyl Sulfoxide ${ }^{1,2}$. Journal of Organic Chemistry, 27, 2377-2383. https://doi.org/10.1021/jo01054a022

[34] Morais, G.R., das Neves Oliveira, M.C. and Thiemann, T. (2006) C7-Substituted Estranes and Related Steroids. Letters of Organic Chemistry, 3, 214-219.

[35] Harada, T., Ikeda, S., Miyazaki, M., Sakata, T. Mori, H. and Matsumura, M. (2007) A Simple Method for Preparing Highly Active Palladium Catalysts Loaded on Various Carbon Supports for Liquid-Phase Oxidation and Hydrogenation Reactions. Journal of Molecular Catalysis A: Chemical, 268, 59-64. https://doi.org/10.1016/j.molcata.2006.12.010

[36] Lu, A.-H., Li, W.-C., Hou, Z. and Schüth, F. (2007) Molecular Level Dispersed Pd Clusters in the Carbon Walls of Ordered Mesoporous Carbon as a Highly Selective Alcohol Oxidation Catalyst. Journal of the Chemical Society, Chemical Communications, 43, 1038-1040. https://doi.org/10.1039/B613834A

[37] Muzart, J. (2006) Molecular Oxygen to Regenerate $\mathrm{Pd}^{\mathrm{II}}$ Active Species. Chemistry-An Asian Journal, 1, 508-515. https://doi.org/10.1002/asia.200600202 